\title{
A smoothed finite element method for plate analysis
}

\author{
H. Nguyen-Xuan* T. Rabczuk ${ }^{\dagger}$ Stéphane Bordas $₫ \S$ J.F.Debongnie $~$
}

September 18, 2007

\begin{abstract}
A quadrilateral element with smoothed curvatures for Mindlin-Reissner plates is proposed. The curvature at each point is obtained by a non-local approximation via a smoothing function. The bending stiffness matrix is calculated by a boundary integral along the boundaries of the smoothing elements (smoothing cells). Numerical results show that the proposed element is robust, computational inexpensive and simultaneously very accurate and free of locking, even for very thin plates. The most promising feature of our elements is their insentivity to mesh distortion.
\end{abstract}

\section{Introduction}

Plate structures play an important role in Engineering Science. There are two different plate theories, the Kirchhoff plate and the Mindlin-Reissner plate theory. Kirchhoff plates are only applicable for thin structures where shear stresses in the plate can be ignored. Moreover, Kirchhoff plate elements require $C^{1}$ continuous shape functions. Mindlin-Reissner plates take shear effects into account. An advantage of the Mindlin-Reissner model over the biharmonic plate model is that the energy involves only first derivatives of the unknowns and so conforming finite element approximations require only the use of $C^{0}$ shape functions instead of the required $C^{1}$ shape functions for the biharmonic model. However, Mindlin-Reissner plate elements exhibit a phenomenon called shear locking when the thickness of the plate tends to zero. Shear locking occurs due to incorrect transverse forces under bending. When linear finite element shape functions are used, the shear angle is linear within an element while the contribution of the displacement is only constant. The linear contribution of the rotation cannot be "balanced" by a contribution from the displacement. Hence, the Kirchhoff constraint $w_{, x}+\beta_{y}=0, w_{, y}+\beta_{x}=0$ is not fulfilled in the entire element any more. Typically, when shear locking occurs, there are large oscillating shear/transverse forces and hence a simple smoothing procedure can drastically improve the results. Early methods tried to overcome the shear locking phenomenon by reduced integration or a selective reduced integration, see References $[63,27,28]$. The idea is to split the strain energy into two parts, one due to bending and the other one due to shear. Commonly, different integration rules for the bending strain and the shear strain energy are used. For example, for the shear strain energy, reduced integration is used while full integration is used for the bending energy. Reduced integration leads to an instability due to rank deficiency and results in zero-energy modes that can be eliminated by an hourglass control, [3,6,26,62].

For a general quadrilateral plate element, the deflection and the two rotations of the four-node element can be interpolated. Often, approximated fields of high degree are used. However, except for the 16-node isoparametric element

\footnotetext{
*Division of Computational Mechanics, Department of Mathematics and Informatics, University of Natural Sciences -VNU-HCM,227 Nguyen Van Cu, Vietnam email: nxhung.hcmuns.edu.vn

†Department of Mechanical Engineering, University of Canterbury, Private Bag 4800, Christchurch 8140, New Zealand email:timon. rabczuk@canterbury.ac.nz

$\ddagger$ University of Glasgow, Civil Engineering, Rankine building, G12 8LT email: stephane.bordas@alumni.northwestern.edu, Tel. +44 (0) 141330 5204, Fax. +44 (0) 1413304557 http://www.civil.gla.ac.uk/ bordas

$\S$ corresponding author

『Division of Manufacturing, University of Liège, Bâtiment B52/3 Chemin des Chevreuils 1, B-4000 Liège 1, Belgium JF. Debongnie@ulg. ac. be
} 
of [62], most other elements still exhibit shear locking when the thickness tends to zero. To overcome this phenomenon, a reduced integration scheme is employed in the shear term. However, most of these elements still exhibit either locking or exhibit artificial zero-energy modes. Another famous class of plate elements are mixed formulation/hybrid elements [35,34, 45] and equilibrium elements [23]. However, such elements are complex and the computational cost is high. They are not popular in most commercial Finite Element Method (FEM) codes.

The Assumed Natural Strain (ANS) method was developed to eliminate shear locking for bilinear plate elements, [29]. The basic idea is to compute the shear strains not directly from the derivatives of the displacements but at discrete collocation points, from the displacements. Afterwards, they are interpolated over the element with specific shape functions. For the bilinear element for example, the collocation points will be placed at the midpoint of the element edges since the shear stresses are linear in the element and zero in the middle of the element. This reduces in addition one of the constraints, since it makes one of the Kirchhoff constraints linear dependent on the other constraints. Bathe and Dvorkin [4] extended the ANS plate elements to shells. The resulting element is known as the MITC (Mixed Interpolation of Tensorial Components) or Bathe-Dvorkin element, see also [22,5]. Many ANS versions of plate and shell elements have been developed. A nice overview can be found e.g in the textbooks by [62] and [3].

An alternative to the ANS method to avoid shear locking is the Discrete-Shear-Gap (DSG) method [10]. The DSG method is in a way similar to the ANS method since it modifies the course of certain strains within the element. The main difference is the lack of collocation points that makes the DSG method independent of the order and form of the element. Instead, the Kirchhoff constraints are imposed directly on the element nodes. The Enhanced-Assumed-Strain (EAS) method principally can be used to avoid locking phenomena as well. While the EAS method was successfully applied to eliminate membrane locking in shells, it was not very efficient in removing shear locking in plates. The EAS element of Simo and Rifai [49] gives satisfying results for rectangular plates.

In this work, we present a new plate elements based on the MITC4 element in which we incorporate stabilized conforming nodal integration (SCNI). The SCNI approach was originally developed in meshfree methods as a normalization for nodal integration $[17,60]$ of the meshfree Galerkin weak form and recently in the FEM [38]. In this approach, the strain smoothing stabilization has been introduced in the SCNI to meet the integration constraints and thus fulfills the linear exactness in the Galerkin approximation of the second order partial differential equations. Wang et al. [52] have shown that the cause of shear locking in Mindlin-Reissner plate formulations is due to the inability of the approximation functions to reproduce the Kirchhoff mode, and the incapability of the numerical method to achieve pure bending exactness (BE) in the Galerkin approximation. In their study, the Kirchhoff mode reproducing condition (KMRC) is ensured for Mindlin-Reissner plates. The approximation functions for the displacement and the rotations are constructed to meet the KMRC. Then, they derived the integration constraints for achieving BE, and a curvature smoothing method (CSM) is proposed to meet the bending integration constraints. A further extension of the SCNI to analysis of transverse and inplane loading of laminated anisotropic plates with general planar geometry [53] is studied. Other contributions to remove shear locking in meshfree plate discretizations are given in [50, 24, 32, 33, 37], and, very recently, $[53,19,2]$.

In mesh-free methods with stabilized nodal integration, the entire domain is discretized into cells defined by the field of nodes, such as the cells of a Voronoï diagram [17,60]. Integration is performed along the edges of each cell. Although meshfree methods such as EFG obtain good accuracy and high convergence rate, the non-polynomial or usually complex approximation space increases the computational cost of numerical integration. Recently, applications of the SCNI to the FEM so-called the smoothed finite element method (SFEM) for two dimensional problems had been proposed by Liu et al. [38,39]. It was shown that the SFEM is stable, accurate and effective. Following the idea of the SFEM, Nguyen et al. [44] has formulated the SFEM with a selective cell-wise smoothing technique in order to eliminate locking in incompressible cases.

We will show by numerical experiments that our element performs better or slightly better than the original MITC4 element, at least for all examples tested. Moreover, due to the integration technique, the element promises to be more accurate especially for distorted meshes. We will also show that our element is free of shear locking.

The outline of the paper is organized as follows. In the next section, we present the basic equations of the plate problem and the weak form. The curvature smoothing stabilization and the finite element discretization using the curvature smoothing method are introduced in Section 3. Several numerical examples are given in Section 4 . Finally, 
Section 5 closes this manuscript with some conclusions and future plans.

\section{Governing equations and weak form}

Let $\Omega$ be the domain in $R^{2}$ occupied by the mid-plane of the plate and $w$ and $\boldsymbol{\beta}=\left(\beta_{x}, \beta_{y}\right)^{T}$ denote the transverse displacement and the rotations in the $x-z$ and $y-z$ planes, see Figure 1, respectively. Assuming that the material is homogeneous and isotropic with Young's modulus $E$ and Poisson's ratio $\nu$, the governing differential equations of the Mindlin-Reissner plate are

$$
\begin{gathered}
-\operatorname{div} \mathbf{D}^{b} \boldsymbol{\kappa}(\boldsymbol{\beta})-\lambda t \boldsymbol{\gamma}(\boldsymbol{\beta})=0 \quad \text { in } \quad \Omega \\
-\lambda t \operatorname{div}(\boldsymbol{\gamma})=p \quad \text { in } \quad \Omega \\
w=\bar{w}, \boldsymbol{\beta}=\overline{\boldsymbol{\beta}} \quad \text { on } \quad \Gamma=\partial \Omega
\end{gathered}
$$

where $t$ is the plate thickness, $p=p(x, y)$ is the transverse loading per unit area, $\lambda=\frac{k E}{2(1+\nu)}, k=5 / 6$ is the shear correction factor and $\mathbf{D}^{b}$ is the tensor of bending moduli, $\kappa$ and $\gamma$ are the bending and shear strains, respectively, defined as

$$
\boldsymbol{\kappa}=\left[\begin{array}{c}
\frac{\partial \beta_{x}}{\partial x} \\
-\frac{\partial \beta_{y}}{\partial y} \\
\frac{\partial \beta_{x}}{\partial y}-\frac{\partial \beta_{y}}{\partial x}
\end{array}\right] \quad, \quad \gamma=\left[\begin{array}{c}
\frac{\partial w}{\partial x}+\beta_{x} \\
\frac{\partial w}{\partial y}-\beta_{y}
\end{array}\right]
$$

The Equations (1) - (3) correspond to the minimization of the total potential function

$$
\Pi_{T P E}=\frac{1}{2} \int_{\Omega} \boldsymbol{\kappa}^{T}: \mathbf{D}^{b}: \boldsymbol{\kappa} d \Omega+\frac{1}{2} \int_{\Omega} \boldsymbol{\gamma}^{T}: \mathbf{D}^{s}: \boldsymbol{\gamma} d \Omega-\int_{\Omega} w p d \Omega
$$

The weak form of the equilibrium equation follows from the stationarity of eq. (5):

$$
\delta \Pi_{T P E}=\int_{\Omega} \delta \boldsymbol{\kappa}^{T}: \mathbf{D}^{b}: \boldsymbol{\kappa} d \Omega+\int_{\Omega} \delta \boldsymbol{\gamma}^{T}: \mathbf{D}^{s}: \boldsymbol{\gamma} d \Omega-\int_{\Omega} \delta w p d \Omega
$$

where the delta denotes the variation. Let us assume that the bounded domain $\Omega$ is discretized into $n_{e}$ finite elements,

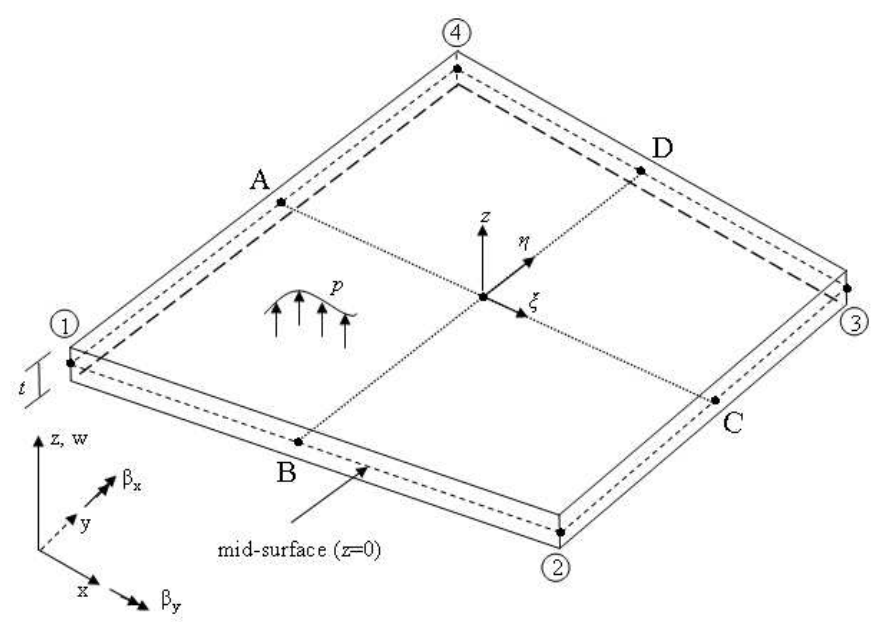

Figure 1: Assumption of shear deformations for quadrilateral plate element 
$\Omega \approx \Omega^{h}=\sum_{e=1}^{n_{e}} \Omega^{e}$. The finite element solution $\mathbf{u}^{h}=\left[w \beta_{x} \beta_{y}\right]^{T}$ of a displacement model for the Mindlin-Reissner plate is then expressed as

$$
\mathbf{u}^{h}=\sum_{i=1}^{n p}\left[\begin{array}{ccc}
N_{i} & 0 & 0 \\
0 & 0 & N_{i} \\
0 & N_{i} & 0
\end{array}\right] \mathbf{q}_{i}
$$

where $n p$ is the total number of element nodes, $N_{i}$ are the bilinear shape functions associated to node $i$ and $\mathbf{q}_{i}=$ $\left[w_{i} \theta_{x i} \theta_{y i}\right]^{T}$ are the nodal degrees of freedom of the variables $\mathbf{u}^{h}=\left[\begin{array}{lll}w & \beta_{x} & \beta_{y}\end{array}\right]^{T}$ associated to node $i$. Then, the discrete curvature field is

$$
\kappa^{h}=\mathbf{B}^{b} \mathbf{q}
$$

where the matrix $\mathbf{B}^{b}$, defined below, contains the derivatives of the shape functions. The approximation of the shear strain is written as

$$
\gamma^{h}=\mathbf{B}^{s} \mathbf{q}
$$

with

$$
\mathbf{B}_{i}^{s}=\left[\begin{array}{ccc}
N_{i, x} & 0 & N_{i} \\
N_{i, y} & -N_{i} & 0
\end{array}\right]
$$

By substituting Equation (7) - Equation (9) into Equation (5) and with the stationarity of (5), we obtain a linear system of an individual element for the vector of nodal unknowns $\mathbf{q}$,

$$
\mathrm{Kq}=\mathrm{g}
$$

with the element stiffness matrix

$$
\mathbf{K}=\int_{\Omega^{e}}\left(\mathbf{B}^{b}\right)^{T} \mathbf{D}^{b} \mathbf{B}^{b} d \Omega+\int_{\Omega^{e}}\left(\mathbf{B}^{s}\right)^{T} \mathbf{D}^{s} \mathbf{B}^{s} d \Omega
$$

and the load vector

$$
\mathbf{g}_{i}=\int_{\Omega^{e}} N_{i}\left[\begin{array}{l}
p \\
0 \\
0
\end{array}\right] d \Omega
$$

where

$$
\mathbf{D}^{b}=\frac{E t^{3}}{12\left(1-\nu^{2}\right)}\left[\begin{array}{ccc}
1 & \nu & 0 \\
\nu & 1 & 0 \\
0 & 0 & \frac{1-\nu}{2}
\end{array}\right] \quad \mathbf{D}^{s}=\frac{E t k}{2(1+\nu)}\left[\begin{array}{ll}
1 & 0 \\
0 & 1
\end{array}\right]
$$

The element stiffness matrix $\mathbf{K}$ is symmetric and positive semi-definite. As already mentioned in the introduction, for a low-order ${ }^{1}$ element, shear locking is observed that can be eliminated by different techniques, $[3,6,26,62]$. The aim of this paper is to propose a stabilized integration for a quadrilateral plate element. Therefore we will

1. apply the curvature smoothing method which was proposed by Chen et al. [17] in meshfree methods based on the nodal integration and recently in the SFEM by Liu et al. [38] to the bending strains and

2. adopt an independent interpolation approximation to the shear strains as the MITC4 element [4].

In mesh-free methods based on nodal integration for Mindlin-Reissner plates, convergence requires fulfilling bending exactness (BE) and thus requires the following bending integration constraint (IC) to be satisfied (Wang et al. [52])

$$
\int_{\Omega} \mathbf{B}_{i}^{b}(\mathbf{x}) d \Omega=\int_{\Gamma} \mathbf{E}_{i}(\mathbf{x}) d \Gamma
$$

\footnotetext{
${ }^{1}$ in our case a four-node quadrilateral full-integrated bilinear finite element
} 
where $\mathbf{B}_{i}$ is the standard gradient matrix

$$
\mathbf{B}_{i}^{b}=\left[\begin{array}{ccc}
0 & 0 & N_{i, x} \\
0 & -N_{i, y} & 0 \\
0 & -N_{i, x} & N_{i, y}
\end{array}\right], \quad \mathbf{E}_{i}=\left[\begin{array}{ccc}
0 & 0 & N_{i} n_{x} \\
0 & -N_{i} n_{y} & 0 \\
0 & -N_{i} n_{x} & N_{i} n_{y}
\end{array}\right]
$$

The IC criterion comes from the equilibrium of the internal and external forces of the Galerkin approximation assuming pure bending (Wang et al. [52]). This is similar to the consistency with the pure bending deformation in the constant moment patch test in FEM.

The basic idea is to couple the MITC element with the curvature smoothing method (CSM). Therefore, smoothing cells are constructed that do not necessarily have to be coincident with the finite elements. We use a mixed variational principle based on an assumed strain field [48] and the integration is carried out either on the elements themselves, or over the smoothing cells that form a partition of the elements. The CSM is employed on each smoothing cell to normalize the local curvature and to calculate the bending stiffness matrix. The shear strains are obtained with independent interpolation functions as in the MITC element.

There are several choices for the smoothing function. For constant smoothing functions, after transforming the volume integral into a surface integral using Gauss' theorem, the surface integration over each smoothing cell becomes a line integration along its boundaries, and consequently, it is unnecessary to compute the gradient of the shape functions to obtain the curvatures and the element bending stiffness matrix. In this paper, we use 1D Gauss integration scheme on all cell edges. The flexibility of the proposed method allows constructing four-node elements even when the elements are extremely distorted [38].

\section{A formulation for four-node plate element}

\subsection{The curvature smoothing method (CSM)}

The CSM was proposed by Chen et al. [17] and Wang et al. [52] as normalization of the local curvature in meshfree methods. A curvature smoothing stabilization is created to compute the nodal curvature by a divergence estimation via a spatial averaging of the curvature fields. In other words, the domain integrals are transformed into boundary integrals. This curvature smoothing avoids the evaluation of the derivatives of the mesh-free shape functions at the nodes $^{2}$, where they vanish, and thus eliminates defective modes. A curvature smoothing at an arbitrary point is given by

$$
\tilde{\boldsymbol{\kappa}}^{h}\left(\mathbf{x}_{C}\right)=\int_{\Omega^{h}} \boldsymbol{\kappa}^{h}(\mathbf{x}) \Phi\left(\mathbf{x}-\mathbf{x}_{C}\right) d \Omega
$$

where $\Phi$ is a smoothing function that has to satisfy the following properties [60]

$$
\Phi \geq 0 \quad \text { and } \quad \int_{\Omega^{h}} \Phi d \Omega=1
$$

For simplicity, $\Phi$ is assumed to be a step function defined by

$$
\Phi\left(\mathbf{x}-\mathbf{x}_{C}\right)=\left\{\begin{array}{l}
1 / A_{C}, \mathbf{x} \in \Omega_{C} \\
0, \mathbf{x} \notin \Omega_{C}
\end{array}\right.
$$

where $A_{C}$ is the area of the smoothing cell, $\Omega_{C} \subset \Omega^{e} \subset \Omega^{h}$, as shown in Figure 2 .

Substituting Equation (19) into Equation (17), and applying the divergence theorem, we obtain

$$
\tilde{\boldsymbol{\kappa}}_{i j}^{h}\left(\mathbf{x}_{C}\right)=\frac{1}{2 A_{C}} \int_{\Omega_{C}}\left(\frac{\partial \theta_{i}^{h}}{\partial x_{j}}+\frac{\partial \theta_{j}^{h}}{\partial x_{i}}\right) d \Omega=\frac{1}{2 A_{C}} \int_{\Gamma_{C}}\left(\theta_{i}^{h} n_{j}+\theta_{j}^{h} n_{i}\right) d \Gamma
$$

\footnotetext{
${ }^{2}$ this applies only to meshfree methods that are based on a nodal integration
} 


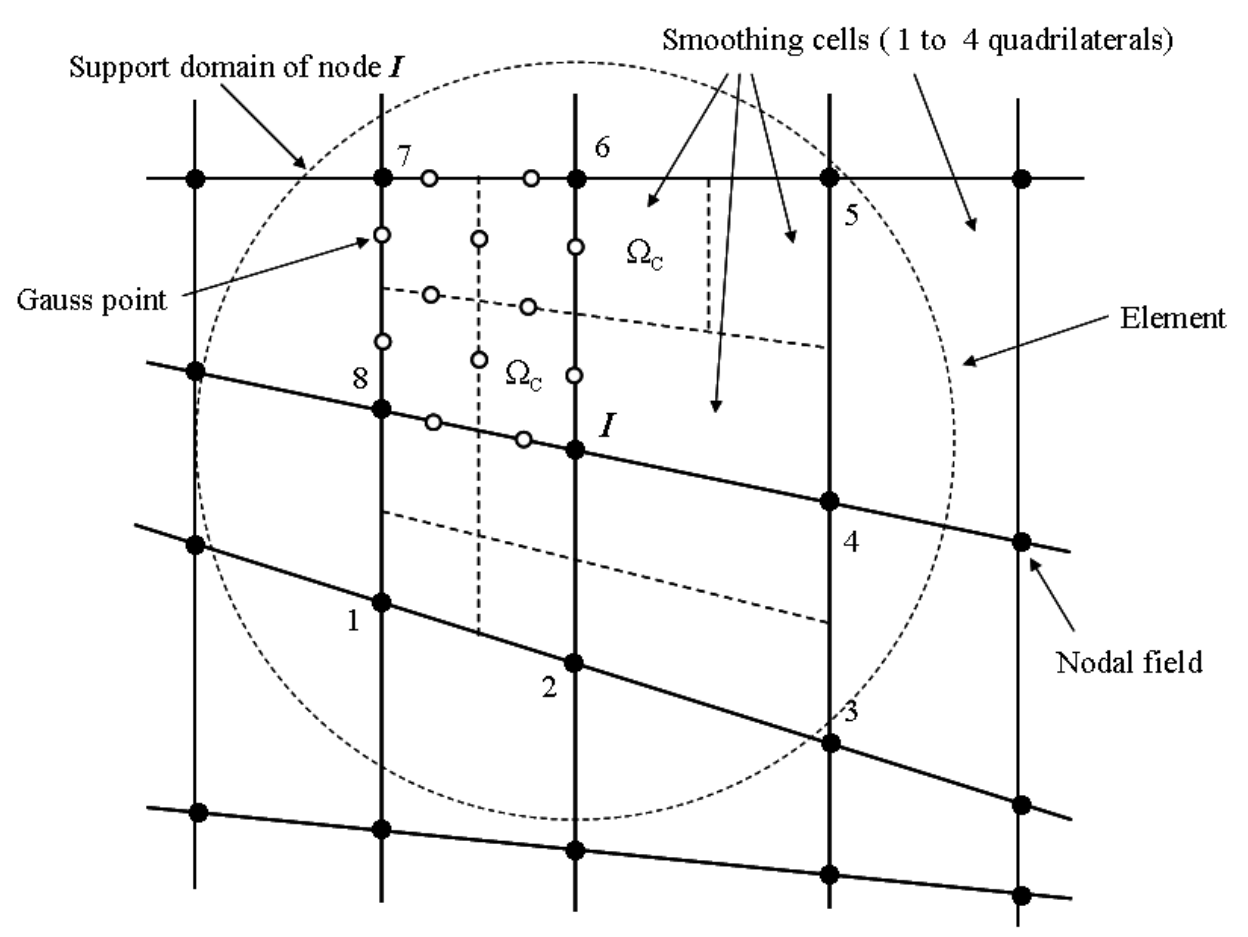

Figure 2: Example of finite element meshes and smoothing cells

Next, we consider an arbitrary smoothing cell, $\Omega_{C}$ illustrated in Figure 2 with boundary $\Gamma_{C}=\bigcup_{b=1}^{n b} \Gamma_{C}^{b}$, where $\Gamma_{C}^{b}$ is the boundary segment of $\Omega_{C}$, and $n b$ is the total number of edges of each smoothing cell. The relationship between the smoothed curvature field and the nodal displacement is written by

$$
\tilde{\boldsymbol{\kappa}}^{h}=\tilde{\mathbf{B}}_{C}^{b} \mathbf{q}
$$

The smoothed element bending stiffness matrix is obtained by

$$
\tilde{\mathbf{K}}^{b}=\int_{\Omega^{e}}\left(\tilde{\mathbf{B}}_{C}^{b}\right)^{T} \mathbf{D}^{b} \tilde{\mathbf{B}}_{C}^{b} d \Omega=\sum_{C=1}^{n c}\left(\tilde{\mathbf{B}}_{C}^{b}\right)^{T}\left(\mathbf{x}_{C}\right) \mathbf{D}^{b} \tilde{\mathbf{B}}_{C}^{b}\left(\mathbf{x}_{C}\right) A_{C}
$$

where $n c$ is the number of smoothing cells of the element, see Figure 3.

Here, the integrands are constant over each $\Omega_{C}$ and the non-local curvature displacement matrix reads

$$
\tilde{B}_{C i}^{b}\left(\mathbf{x}_{C}\right)=\frac{1}{A_{C}} \int_{\Gamma_{C}}\left(\begin{array}{ccc}
0 & 0 & N_{i} n_{x} \\
0 & -N_{i} n_{y} & 0 \\
0 & -N_{i} n_{x} & N_{i} n_{y}
\end{array}\right) d \Gamma
$$

We use Gauss quadrature to evaluate (23) with one integration point over each line segment $\Gamma_{C}^{b}$ :

$$
\tilde{B}_{C i}^{b}\left(\mathbf{x}_{C}\right)=\frac{1}{A_{C}} \sum_{b=1}^{n b}\left(\begin{array}{ccc}
0 & 0 & N_{i}\left(\mathbf{x}_{b}^{G}\right) n_{x} \\
0 & -N_{i}\left(\mathbf{x}_{b}^{G}\right) n_{y} & 0 \\
0 & -N_{i}\left(\mathbf{x}_{b}^{G}\right) n_{x} & N_{i}\left(\mathbf{x}_{b}^{G}\right) n_{y}
\end{array}\right) l_{b}^{C}
$$

where $\mathbf{x}_{b}^{G}$ and $l_{b}^{C}$ are the midpoint (Gauss point) and the length of $\Gamma_{b}^{C}$, respectively. 
The smoothed curvatures lead to high flexibility such as arbitrary polygonal elements, and a slight reduction in computational cost. The element is subdivided into nc non-overlapping sub-domains also called smoothing cells. Figure 3 illustrates different smoothing cells for $\mathrm{nc}=1,2,3$ and 4 corresponding to 1-subcell, 2-subcell, 3-subcell and 4-subcell methods. The curvature is smoothed over each sub-cell. The values of the shape functions are indicated at the corner nodes in Figure 3 in the format $\left(N_{1}, N_{2}, N_{3}, N_{4}\right)$. The values of the shape functions at the integration nodes are determined based on the linear interpolation of shape functions along boundaries of the element or the smoothing cells.

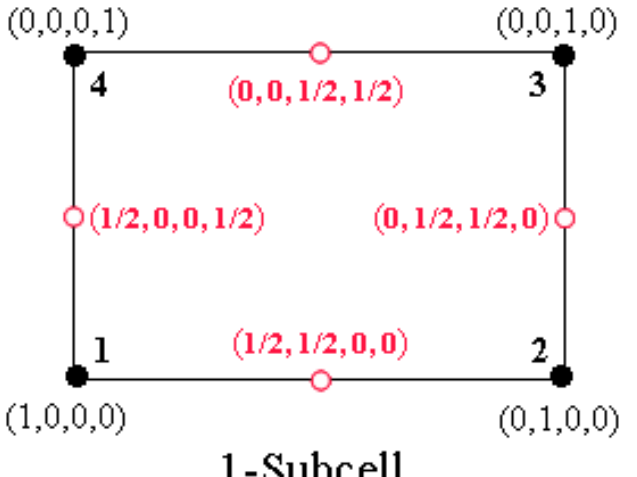

1-Subcell

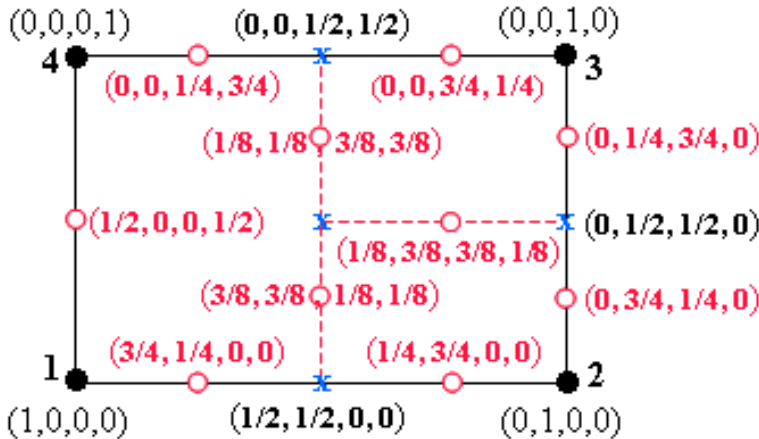

3-Subcell

Field node

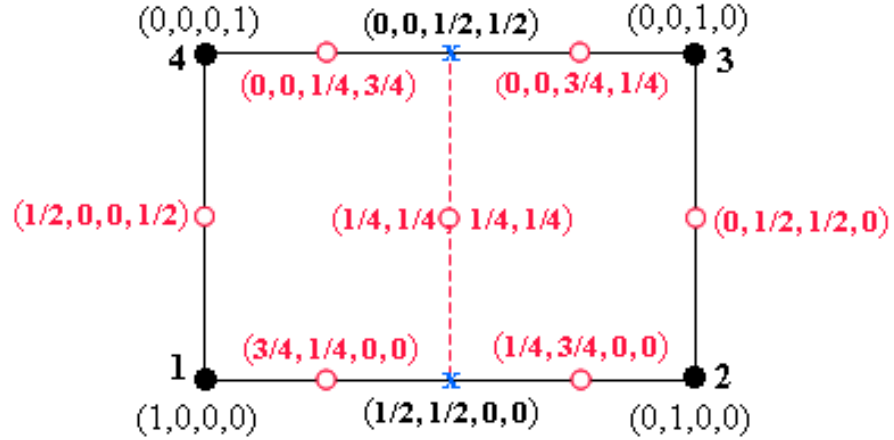

2-Subcell

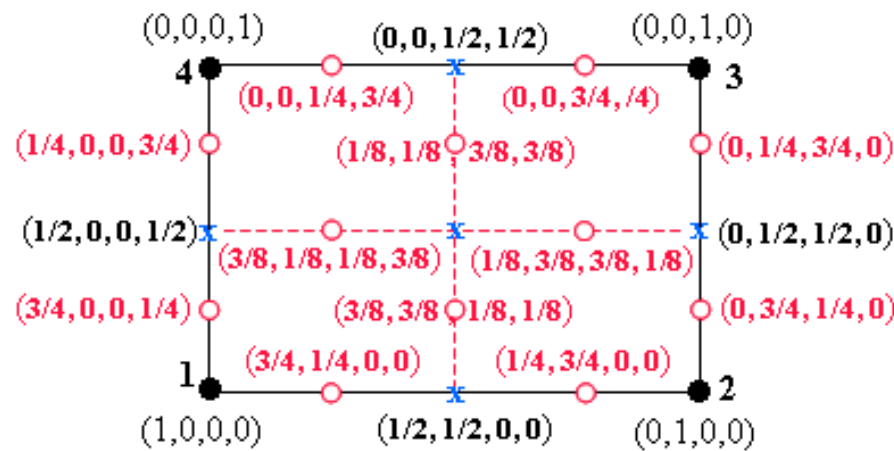

4-Subcell

Integration node

Figure 3: Division of an element into smoothing cells (nc) and the value of the shape function along the boundaries of cells: $\mathrm{k}$-Subcell stands for the shape function of the MISCk element, $k=1,2,3,4$

Therefore the element stiffness matrix in (12) can be modified as follows:

$$
\tilde{\mathbf{K}}=\tilde{\mathbf{K}}^{b}+\mathbf{K}^{s}=\sum_{C=1}^{n c}\left(\tilde{\mathbf{B}}_{C}^{b}\right)^{T} \mathbf{D}^{b} \tilde{\mathbf{B}}_{C}^{b} A_{C}+\int_{\Omega^{e}}\left(\mathbf{B}^{s}\right)^{T} \mathbf{D}^{s} \mathbf{B}^{s} d \Omega
$$

It can be seen that a reduced integration on the shear term $\mathbf{K}^{s}$ is necessary to avoid shear locking. We will denote these elements by SC1Q4, SC2Q4, SC3Q4 and SC4Q4 corresponding to subdivision into $n c=1,2,3$ and 4 smoothing 
cells, Figure 3. However, we will show that these elements fail the patch test and they exhibit an instability due to rank deficiency. Therefore, we employ a mixed interpolation as in the MITC4 element and use independent interpolation fields in the natural coordinate system [4] for the approximation of the shear strains:

$$
\left[\begin{array}{l}
\gamma_{x} \\
\gamma_{y}
\end{array}\right]=\mathbf{J}^{-1}\left[\begin{array}{l}
\gamma_{\xi} \\
\gamma_{\eta}
\end{array}\right]
$$

where

$$
\gamma_{\xi}=\frac{1}{2}\left[(1-\eta) \gamma_{\xi}^{B}+(1+\eta) \gamma_{\xi}^{D}\right], \gamma_{\eta}=\frac{1}{2}\left[(1-\xi) \gamma_{\eta}^{A}+(1+\xi) \gamma_{\eta}^{C}\right]
$$

where $\mathbf{J}$ is the Jacobian matrix and the midside nodes A, B, C, D are shown in Figure 1. Presenting $\gamma_{\xi}^{B}, \gamma_{\xi}^{D}$ and $\gamma_{\eta}^{A}, \gamma_{\eta}^{C}$ based on the discretized fields $u^{h}$, we obtain the shear matrix:

$$
\mathbf{B}_{i}^{s}=\mathbf{J}^{-1}\left[\begin{array}{lll}
N_{i, \xi} & -b_{i}^{12} N_{i, \xi} & b_{i}^{11} N_{i, \xi} \\
N_{i, \eta} & -b_{i}^{22} N_{i, \eta} & b_{i}^{21} N_{i, \eta}
\end{array}\right]
$$

where

$$
b_{i}^{11}=\xi_{i} x_{, \xi}^{M}, \quad b_{i}^{12}=\xi_{i} y_{, \xi}^{M}, \quad b_{i}^{21}=\eta_{i} x_{, \eta}^{L}, \quad b_{i}^{22}=\eta_{i} y_{, \eta}^{L}
$$

with $\xi_{i} \in\{-1,1,1,-1\}, \quad \eta_{i} \in\{-1,-1,1,1\}$ and $(i, M, L) \in\{(1, B, A) ;(2, B, C) ;(3, D, C) ;(4, D, A)\}$. Note that the shear term $\mathbf{K}^{s}$ is still computed by $2 \times 2$ Gauss quadrature while the element bending stiffness $\mathbf{K}^{b}$ in Equation (12) is replaced by the smoothed curvature technique on each smoothing cell of the element.

\section{2 $\mathrm{Hu}-$ Washizu variational formulation}

We use a modified $\mathrm{Hu}$-Washizu variational formulation [54] given for an individual element by

$$
\begin{gathered}
\Pi_{H W}^{e}(w, \tilde{\boldsymbol{\kappa}}, \mathbf{M})= \\
\frac{1}{2} \int_{\Omega^{e}} \tilde{\boldsymbol{\kappa}}^{T}: \mathbf{D}^{b}: \tilde{\boldsymbol{\kappa}} d \Omega-\int_{\Omega^{e}} \mathbf{M}:(\tilde{\boldsymbol{\kappa}}-\boldsymbol{\kappa}) d \Omega+\frac{1}{2} \int_{\Omega^{e}} \boldsymbol{\gamma}^{T}: \mathbf{D}^{s}: \boldsymbol{\gamma} d \Omega-\int_{\Omega^{e}} w p d \Omega
\end{gathered}
$$

where $\mathbf{M}$ is the moment tensor. Partitioning the element into $n c$ sub-cells such that the sub-cells are not overlapping and form a partition of the element $\Omega^{e}, \Omega^{e}=\bigcup_{i c=1}^{n c} \Omega_{i c}^{e}$, the functional energy, $\Pi_{H W}^{e}$, can be rewritten as

$$
\begin{gathered}
\Pi_{H W}^{e}(w, \tilde{\boldsymbol{\kappa}}, \mathbf{M})= \\
\frac{1}{2} \sum_{i c=1}^{n c} \int_{\Omega_{i c}^{e}} \tilde{\boldsymbol{\kappa}}_{i c}: \mathbf{D}^{b}: \tilde{\boldsymbol{\kappa}}_{i c} d \Omega_{i c}^{e}-\sum_{i c=1}^{n c} \int_{\Omega_{i c}^{e}} \mathbf{M}:(\tilde{\boldsymbol{\kappa}}-\boldsymbol{\kappa}) d \Omega_{i c}^{e}+\frac{1}{2} \int_{\Omega^{e}} \boldsymbol{\gamma}: \mathbf{D}^{s}: \boldsymbol{\gamma} d \Omega-\int_{\Omega^{e}} w p d \Omega
\end{gathered}
$$

where

$$
\tilde{\boldsymbol{\kappa}}_{i c}=\frac{1}{A_{i c}} \int_{\Omega_{i c}^{e}} \boldsymbol{\kappa}(\mathbf{x}) d \Omega_{i c}^{e} \text { and } A^{e}=\sum_{i c=1}^{n c} A_{i c}
$$

with $A_{i c}$ is the area of the smoothing cell, $\Omega_{i c}^{e}$.

To reduce $\Pi_{H W}^{e}$ from a three-field potential to a two-field potential, we need to find a strict condition on the smoothing cells $\Omega_{C}$ for the orthogonality condition $[48,49]$ :

$$
\int_{\Omega^{e}} \mathbf{M}: \hat{\boldsymbol{\kappa}} d \Omega=\int_{\Omega^{e}} \mathbf{M}:(\tilde{\boldsymbol{\kappa}}-\boldsymbol{\kappa}) d \Omega=0
$$

is satisfied. By substituting $\mathbf{M}$ through the constitutive relation $\mathbf{M}=\mathbf{D}^{b} \tilde{\boldsymbol{\kappa}}$, we rewrite the orthogonality condition: 


$$
\begin{gathered}
\int_{\Omega^{e}} \mathbf{M}: \hat{\boldsymbol{\kappa}} d \Omega=\sum_{i c=1}^{n c} \int_{\Omega_{i c}^{e}} \mathbf{D}^{b} \tilde{\boldsymbol{\kappa}}:(\tilde{\boldsymbol{\kappa}}-\boldsymbol{\kappa}) d \Omega_{i c}^{e}=\sum_{i c=1}^{n c} \int_{\Omega_{i c}^{e}} \mathbf{D}^{b} \tilde{\boldsymbol{\kappa}}:\left(\frac{1}{A_{C}} \int_{\Omega_{C}} \boldsymbol{\kappa} d \Omega_{C}-\boldsymbol{\kappa}\right) d \Omega_{i c}^{e} \\
=\sum_{i c=1}^{n c} \mathbf{D}^{b} \tilde{\boldsymbol{\kappa}}: \int_{\Omega_{i c}^{e}}\left(\frac{1}{A_{C}} \int_{\Omega_{C}} \boldsymbol{\kappa} d \Omega_{C}-\boldsymbol{\kappa}\right) d \Omega_{i c}^{e}=\sum_{i c=1}^{n c} \mathbf{D}^{b} \tilde{\boldsymbol{\kappa}}:\left(\frac{\int_{\Omega_{i c}^{e}} d \Omega_{i c}^{e}}{A_{C}} \int_{\Omega_{C}} \boldsymbol{\kappa} d \Omega_{C}-\int_{\Omega_{i c}^{e}} \boldsymbol{\kappa} d \Omega_{i c}^{e}\right) \\
=\sum_{i c=1}^{n c} \mathbf{D}^{b} \tilde{\boldsymbol{\kappa}}:\left(\frac{A_{i c}}{A_{C}} \int_{\Omega_{C}} \boldsymbol{\kappa} d \Omega_{C}-\int_{\Omega_{i c}^{e}} \boldsymbol{\kappa} d \Omega_{i c}^{e}\right)
\end{gathered}
$$

where $\Omega_{C} \subset \Omega^{e}$ is a smoothed curvature field defined for every $\tilde{\boldsymbol{\kappa}}=\frac{1}{A_{C}} \int_{\Omega_{C}} \boldsymbol{\kappa}(\mathbf{x}) d \Omega$ and the smoothed curvature $\tilde{\boldsymbol{\kappa}}$ does not depend on the integration after processing a smoothed operator, i.e, $\int_{\Omega_{i c}^{e}} \mathbf{D}^{b} \tilde{\boldsymbol{\kappa}} d \Omega_{i e}^{e}=\mathbf{D}^{b} \tilde{\boldsymbol{\kappa}} A_{i c}$ with

$$
A_{C}=A_{i c} \text { and } \Omega_{C} \equiv \Omega_{i c}^{e}
$$

If $\Omega_{C}$ coincides with $\Omega_{i c}^{e}$, the orthogonality condition (33) is met and the three-field potential is reduced to a two-field potential:

$$
\Pi_{H W}^{e}(w, \tilde{\boldsymbol{\kappa}})=\frac{1}{2} \sum_{i c=1}^{n c} \int_{\Omega_{i c}^{e}} \tilde{\boldsymbol{\kappa}}_{i c}: \mathbf{D}^{b}: \tilde{\boldsymbol{\kappa}}_{i c} d \Omega_{i c}^{e}+\frac{1}{2} \int_{\Omega^{e}} \gamma: \mathbf{D}^{s}: \gamma d \Omega-\int_{\Omega^{e}} w p d \Omega
$$

Now we show that the proposed total energy approaches the total potential energy variational principle (TPE) when $n c$ tends to infinity. Based on the definition of the double integral formula, when $n c \rightarrow \infty, A_{i c} \rightarrow d A_{i c}-$ an infinitesimal area containing point $\mathbf{x}_{i c}$, applying the mean value theorem for the smoothed strain,

$$
\tilde{\boldsymbol{\kappa}}_{i c}=\int_{\Omega_{i c}^{e}} \frac{\boldsymbol{\kappa}(\mathbf{x})}{A_{i c}} d \Omega_{i c}^{e} \longrightarrow \boldsymbol{\kappa}\left(\mathbf{x}_{i c}\right)
$$

where $\boldsymbol{\kappa}(\mathbf{x})$ is assumed to be a continuous function. Equation (37) states that the average value of $\boldsymbol{\kappa}(\mathbf{x})$ over a domain $\Omega_{i c}^{e}$ approaches its value at the converged point $\mathbf{x}_{i c}$.

Taking the limit of $\Pi_{H W}^{e}$ when the number of subcells tends to infinity,

$$
\begin{gathered}
\lim _{n c \rightarrow \infty} \Pi_{H W}^{e}(w, \tilde{\boldsymbol{\kappa}})= \\
\frac{1}{2} \lim _{n c \rightarrow \infty} \sum_{i c=1}^{n c} \int_{\Omega_{i c}^{e}} \mathbf{D}^{b}:\left(\int_{\Omega_{i c}^{e}} \frac{\boldsymbol{\kappa}(\mathbf{x})}{A_{i c}} d \Omega_{i c}^{e}\right):\left(\int_{\Omega_{i c}^{e}} \frac{\boldsymbol{\kappa}(\mathbf{x})}{A_{i c}} d \Omega_{i c}^{e}\right) d \Omega_{i c}^{e}+\frac{1}{2} \int_{\Omega^{e}} \boldsymbol{\gamma}: \mathbf{D}^{s}: \boldsymbol{\gamma} d \Omega-\int_{\Omega^{e}} w p d \Omega \\
=\frac{1}{2} \lim _{n c \rightarrow \infty} \sum_{i c=1}^{n c} \mathbf{D}^{b}: \boldsymbol{\kappa}\left(\mathbf{x}_{i c}\right): \boldsymbol{\kappa}\left(\mathbf{x}_{i c}\right) d A_{i c}+\frac{1}{2} \int_{\Omega^{e}} \boldsymbol{\gamma}: \mathbf{D}^{s}: \boldsymbol{\gamma} d \Omega-\int_{\Omega^{e}} w p d \Omega \\
=\frac{1}{2} \int_{\Omega^{e}} \mathbf{D}^{b}: \boldsymbol{\kappa}(\mathbf{x}): \boldsymbol{\kappa}(\mathbf{x}) d \Omega+\frac{1}{2} \int_{\Omega^{e}} \gamma: \mathbf{D}^{s}: \boldsymbol{\gamma} d \Omega-\int_{\Omega^{e}} w p d \Omega=\Pi_{T P E}^{e}(w, \boldsymbol{\beta})
\end{gathered}
$$

The above proves that the total potential energy variational principle is recovered from the proposed variational formulation as $n c$ tends to infinity.

\section{Numerical results}

We will test our new element for different numbers of smoothing cells and call our element MISCk (Mixed Interpolation and Smoothed Curvatures) with $\mathrm{k} \in\{1,2,3,4\}$ smoothing cells for the bending terms. For instance, the MISC1 element is the element with only one smoothing cell to integrate the bending part of the element stiffness matrix. We will compare our results to the results obtained with the reduced/selective integrated quadrilateral element (Q4-R), the MITC4 element and with several other elements in the literatures. 


\subsection{Patch test}

The patch test was introduced by Bruce Irons and Bazeley [7] to check the convergence of finite elements. It is checked if the element is able to reproduce a constant distribution of all quantities for arbitrary meshes. It is important that one element is completely surrounded by neighboring elements in order to test if a rigid body motion is modelled correctly, Figure 4. The boundary deflection is assumed to be $w=\frac{1}{2}\left(1+x+2 y+x^{2}+x y+y^{2}\right)[18]$. The results are shown in Table 1. While the MITC4 element and the MISCk elements pass the patch test, the Q4-R element and the SC1Q4, SC2Q4, SC3Q4, SC4Q4 elements fail the patch test. Note that also the fully integrated Q4 element (on both the bending and the shear terms) does not pass the patch test.

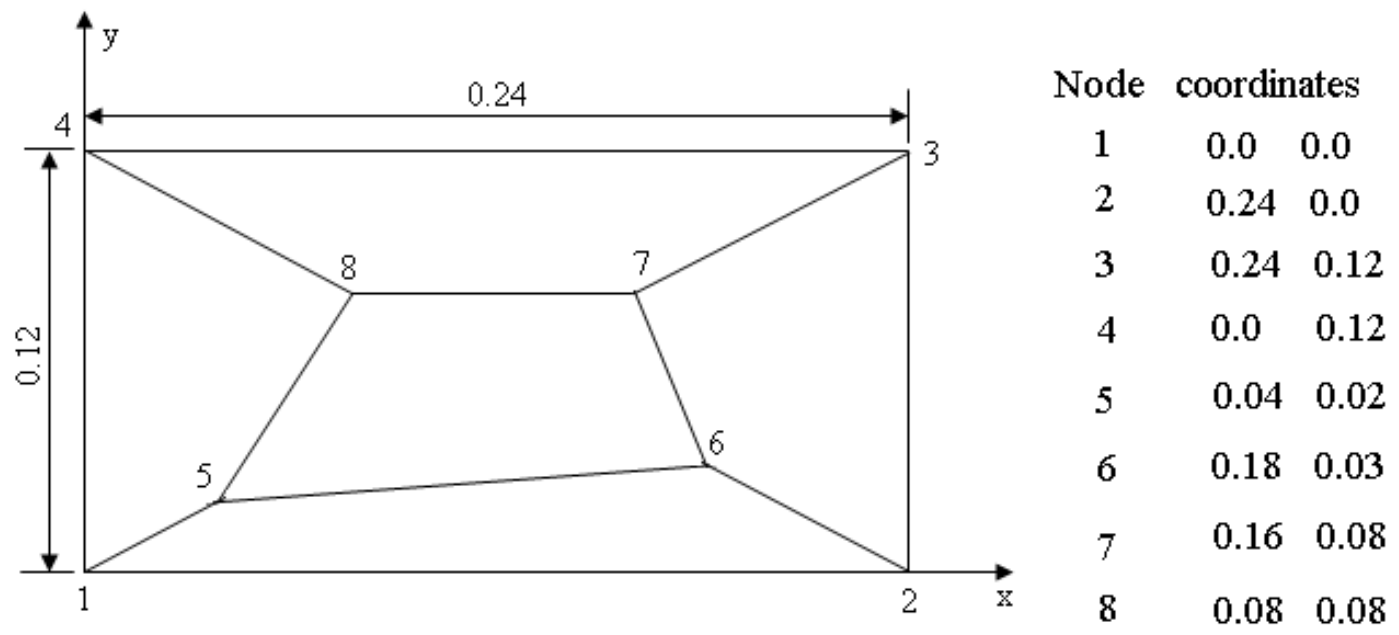

Figure 4: Patch test of elements

Table 1: Patch test

\begin{tabular}{ccccccc}
\hline Element & $w_{5}$ & $\theta_{x 5}$ & $\theta_{y 5}$ & $m_{x 5}$ & $m_{y 5}$ & $m_{x y 5}$ \\
\hline Q4-R & 0.5440 & 1.0358 & -0.676 & - & - & - \\
SC1Q4 & 0.5431 & 1.0568 & -0.7314 & - & - & - \\
SC2Q4 & 0.5439 & 1.0404 & -0.6767 & - & - & - \\
SC3Q4 & 0.5440 & 1.0396 & -0.6784 & - & - & - \\
SC4Q4 & 0.5439 & 1.0390 & -0.6804 & - & - & - \\
MITC4 & 0.5414 & 1.04 & -0.55 & -0.01111 & -0.01111 & -0.00333 \\
MISC1 & 0.5414 & 1.04 & -0.55 & -0.01111 & -0.01111 & -0.00333 \\
MISC2 & 0.5414 & 1.04 & -0.55 & -0.01111 & -0.01111 & -0.00333 \\
MISC3 & 0.5414 & 1.04 & -0.55 & -0.01111 & -0.01111 & -0.00333 \\
MISC4 & 0.5414 & 1.04 & -0.55 & -0.01111 & -0.01111 & -0.00333 \\
Exact & 0.5414 & 1.04 & -0.55 & -0.01111 & -0.01111 & -0.00333 \\
\hline
\end{tabular}




\subsection{A sensitivity test of mesh distortion}

Consider a clamped square plate subjected to a center point $F$ or uniform load $p$ shown in Figure 5 . The geometry parameters and the Poisson's ratio are: length $L$, thickness $t$, and $\nu=0.3$. Due to its symmetry, only a quarter (lower - left) of the plate is modelled with a mesh of $8 \times 8$ elements. To study the effect of mesh distortion on the results, interior nodes are moved by an irregularity factor $s$. The coordinates of interior nodes is perturbed as follows [38]:

$$
\begin{aligned}
& x^{\prime}=x+r_{c} s \Delta x \\
& y^{\prime}=y+r_{c} s \Delta y
\end{aligned}
$$

where $r_{c}$ is a generated random number given values between -1.0 and 1.0,s $\in[0,0.5]$ is used to control the shapes of the distorted elements and $\Delta x, \Delta y$ are initial regular element sizes in the $x$-and $y$-directions, respectively.

For the concentrated center point load $F$, the influence of the mesh distortion on the center deflection is given in Figure 6 for a thickness ratio of $(t / L=0.01$ and 0.001$)$. The results of our presented method are more accurate than those of the Q4-R element and the MITC4 element, especially for extremely distorted meshes. Here, the MISC1 element gives the best result. However, this element contains two zero-energy modes. In simple problems, these hourglass modes can be automatically eliminated by the boundary conditions. However, this is not in general the case. Otherwise, the MISC2, MISC3 and MISC4 elements retain a sufficient rank of the element stiffness matrix and give excellent results.

Let us consider a thin plate with $(t / L=0.001)$ under uniform load as shown Figure 5 a. The numerical results of the central deflections are shown in Table 2 and Figure 7 and compared to other elements. For the case $s=0$, it can be seen that the MISCk elements are the same or slightly more accurate results than the other elements. Moreover, all proposed elements are better than the comparison elements especially for distorted meshes, $s>0$.

Table 2: The central deflection $w_{c} /\left(p L^{4} / 100 D\right), D=E t^{3} / 12\left(1-\nu^{2}\right)$ with mesh distortion for thin clamped plate subjected to uniform load $p$

\begin{tabular}{cccccccc}
\hline s & -1.249 & -1.00 & -0.5 & 0.00 & 0.5 & 1.00 & 1.249 \\
\hline CRB1 [55] & 0.1381 & 0.1390 & 0.1247 & 0.1212 & 0.1347 & 0.1347 & 0.1249 \\
CRB2 [55] & 0.2423 & 0.1935 & 0.1284 & 0.1212 & 0.1331 & 0.1647 & 0.1947 \\
S1 [55] & 0.1105 & 0.1160 & 0.1209 & 0.1211 & 0.1165 & 0.1059 & 0.0975 \\
S4R [1] & 0.1337 & 0.1369 & 0.1354 & 0.1295 & 0.1234 & 0.1192 & 0.1180 \\
DKQ [31] & 0.1694 & 0.1658 & 0.1543 & 0.1460 & 0.1418 & 0.1427 & 0.1398 \\
ANS-EC [30] & - & - & - & 0.1303 & - & - & - \\
ANS-2ND [30] & - & - & - & 0.1240 & - & - & - \\
Q4BL [61] & - & - & - & 0.1113 & - & - & - \\
NCQ [40] & - & - & - & 0.1278 & - & - & - \\
MITC4 & 0.0973 & 0.1032 & 0.1133 & 0.1211 & 0.1245 & 0.1189 & 0.1087 \\
MISC1 & 0.1187 & 0.1198 & 0.1241 & 0.1302 & 0.1361 & 0.1377 & 0.1347 \\
MISC2 & 0.1151 & 0.1164 & 0.1207 & 0.1266 & 0.1323 & 0.1331 & 0.1287 \\
MISC3 & 0.1126 & 0.1144 & 0.1189 & 0.1249 & 0.1305 & 0.1309 & 0.1260 \\
MISC4 & 0.1113 & 0.1130 & 0.1174 & 0.1233 & 0.1287 & 0.1288 & 0.1227 \\
Exact solu. & 0.1265 & 0.1265 & 0.1265 & 0.1265 & 0.1265 & 0.1265 & 0.1265 \\
\hline
\end{tabular}

\subsection{Square plate subjected to a uniform load or a point load}

Figure 5a and Figure 13 are the model of a square plate with clamped and simply supported boundary conditions, respectively, subjected to a uniform load $p=1$ or a central load $F=16.3527$. The material parameters are given 


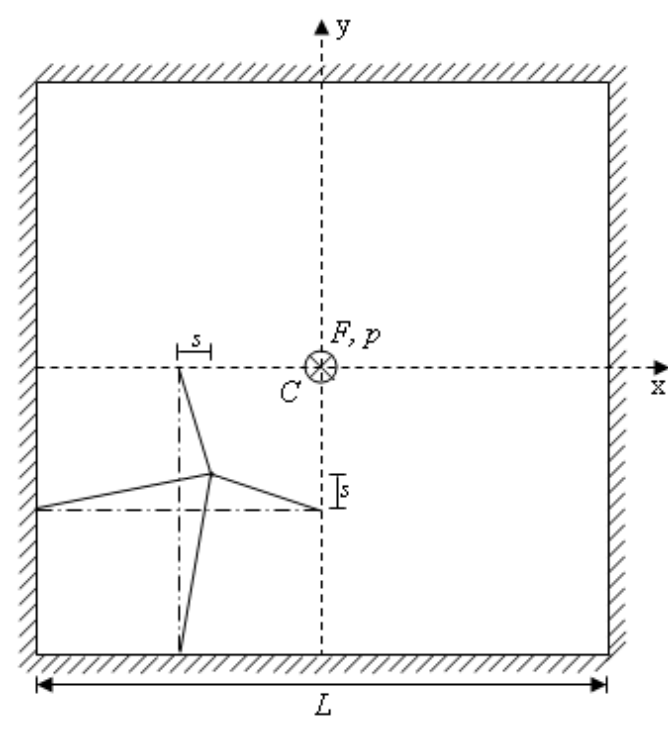

(a)

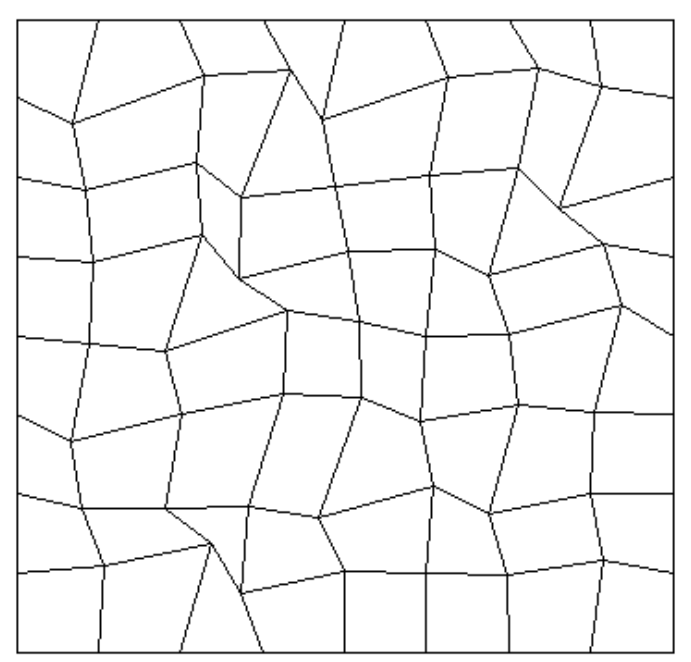

(c)

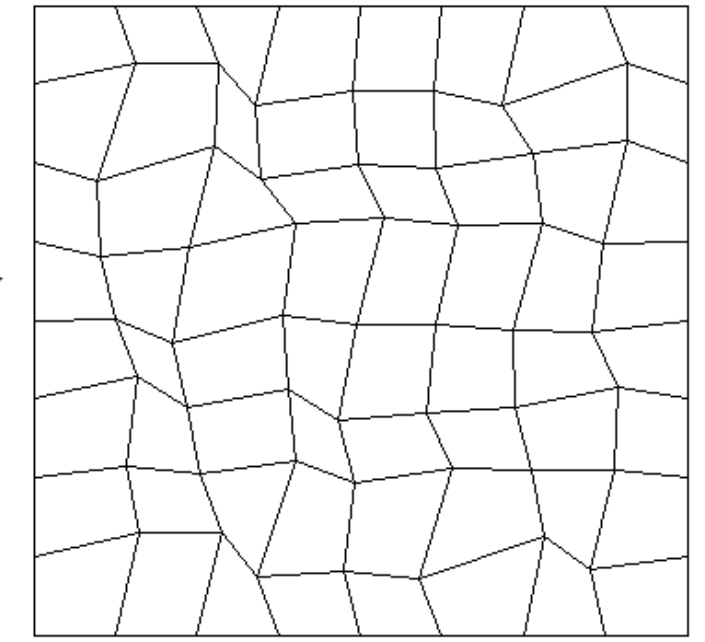

(b)

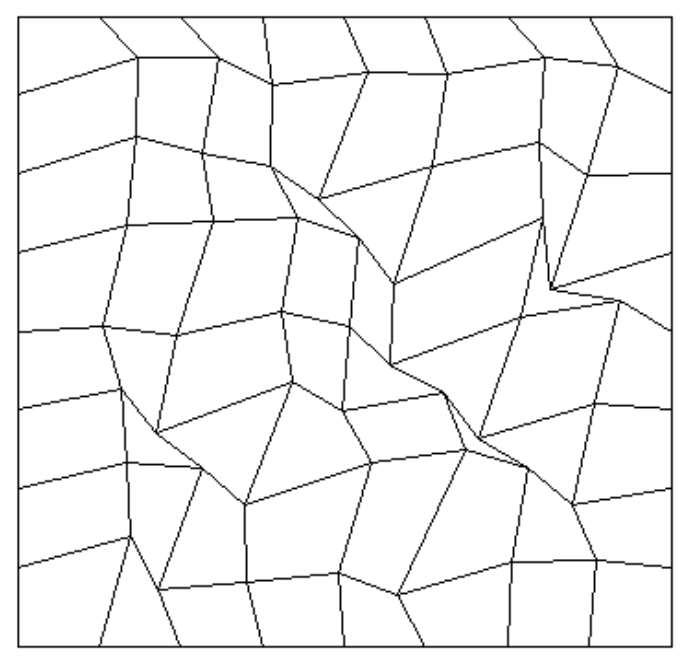

(d)

Figure 5: Effect of mesh distortion for a clamped square plate: (a) clamped plate model; (b) $s=0.3$; (c) $s=0.4$; and (d) $s=0.5$ 


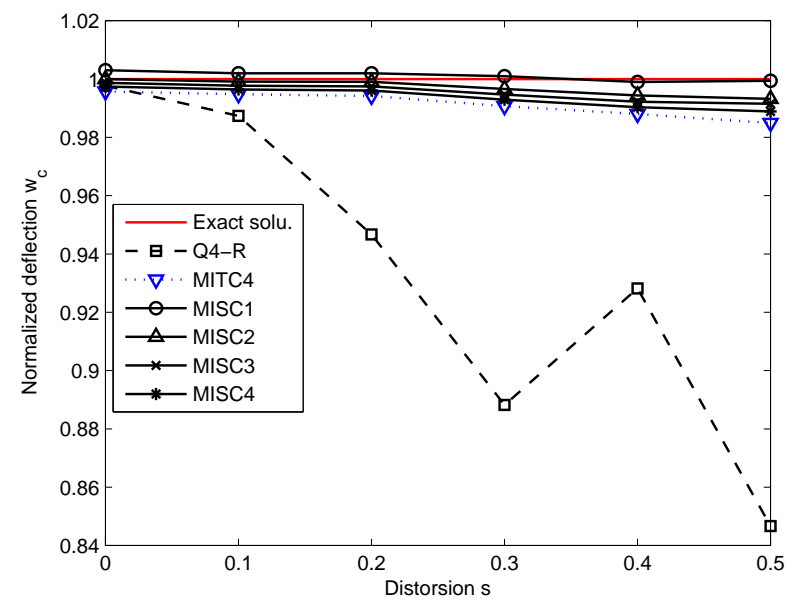

(a)

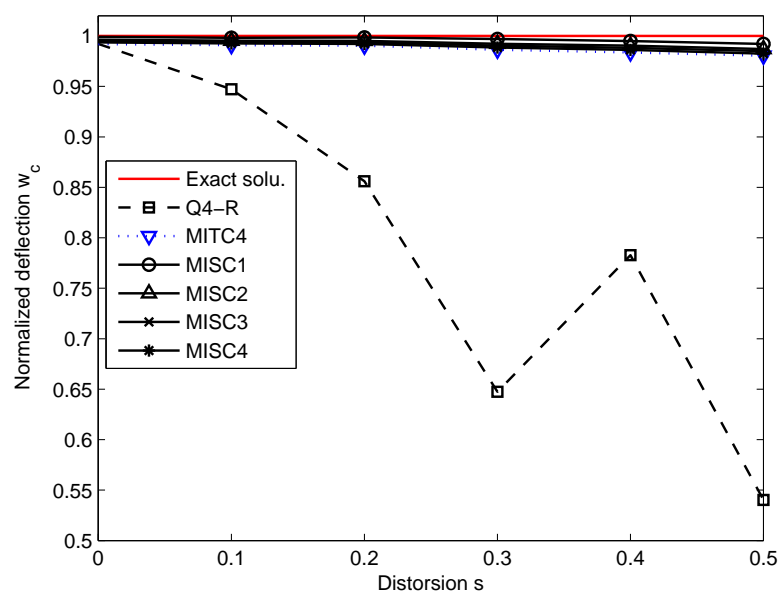

(b)

Figure 6: The normalized center deflection with influence of mesh distortion for a clamped square plate subjected to a concentrated load: a) $\mathrm{t} / \mathrm{L}=0.01$, b) $\mathrm{t} / \mathrm{L}=0.001$

by Young's modulus $E=1092000$ and Poisson's ratio $\nu=0.3$. Uniform meshes with $N=2,4,8,16,32$ are used and symmetry conditions are exploited.

For a clamped case, Figure 8 illustrates the convergence of the normalized deflection and the normalized moment at the center versus the mesh density $N$ for a relation $t / L=0.01$. Even for very coarse meshes, the deflection tends to the exact solution. For the finest mesh, the displacement slightly $(.06 \%)$ exceeds the value of the exact solution. The bending moment converges to the analytical value. The rate of convergence in the energy norm is presented in Figure 9 and is for all elements equal to 1.1 but the MISCk elements are more accurate than the MITC4 element in energy.

Tables 3-4 show the performance of the plate element for different thickness ratios, $t / L=10^{-1} \sim 10^{-5}$. No shear locking is observed. In addition, it is observed that the MISCk elements improve the solutions with coarse meshes while for fine meshes all elements have almost marginal differences.

Next we consider a sequence of distorted meshes with 25, 81, 289 and 1089 nodes as shown in Figure 10. The numerical results in terms of the error in the central displacement and the strain energy are illustrated in Figure 11. All proposed elements give stable and accurate results. Especially for coarse meshes, the MISCk elements are more accurate than the MITC4 element; a reason for this may be that for our finest meshes, fewer elements are distorted in comparison to coarse meshes.

Now we will test the computing time for the clamped plate analyzed above. The program is compiled by a personal computer with Pentium(R)4, CPU-3.2GHz and RAM-512MB. The computational cost to set up the global stiffness matrix and to solve the algebraic equations is illustrated in Figure 12. The MISCk elements and the MITC4 element give nearly the same CPU time for coarse meshes where the MISCk elements are more accurate. From the plots, we can conjecture that for finer meshes, the MITC4 element is computationally more expensive than the MISCk element, and the MISCk elements are generally more accurate. The lower computational cost comes from the fact that no computation of the Jacobian matrix is necessary for the MISCk elements while the MITC4 element needs to determine the Jacobian determinant, the inverse of the Jacobian matrix (transformation of two coordinates; global coordinate and local coordinate) and then the stiffness matrix is calculated by $2 \times 2$ Gauss points. Previously, the same tendency was observed for the standard (Q4 element), see [38] for details.

For a simply supported plate subjected to central concentrate load, the same tendencies as described above are 


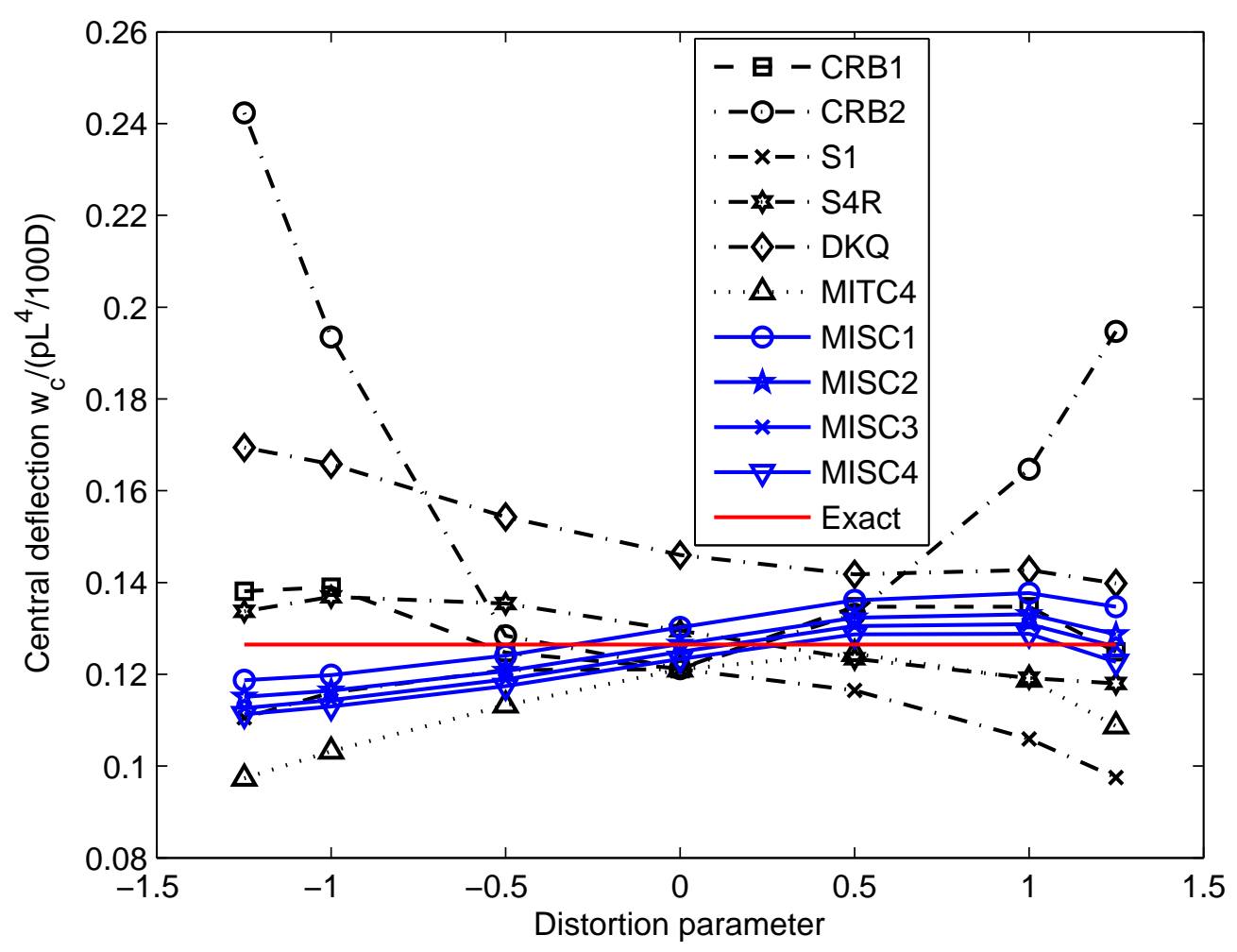

Figure 7: Comparison of other elements through the center deflection with mesh distortion

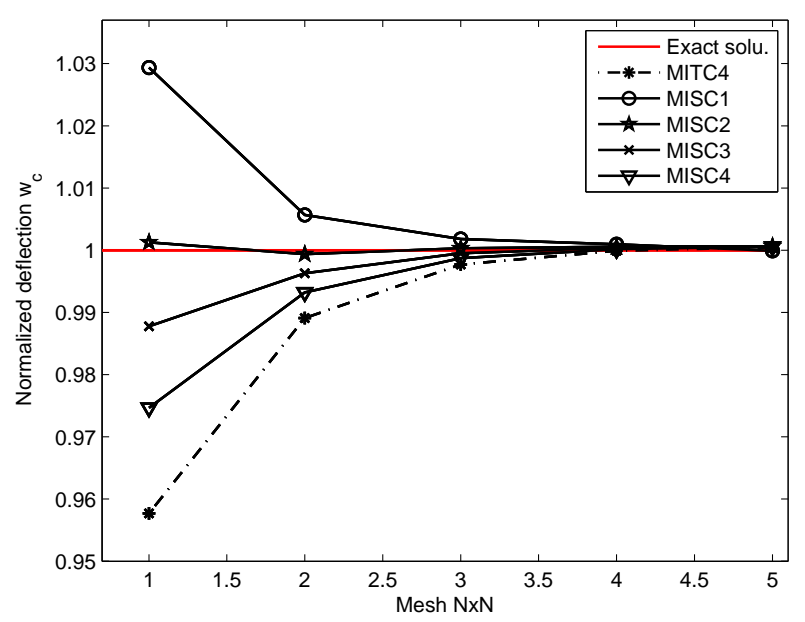

(a)

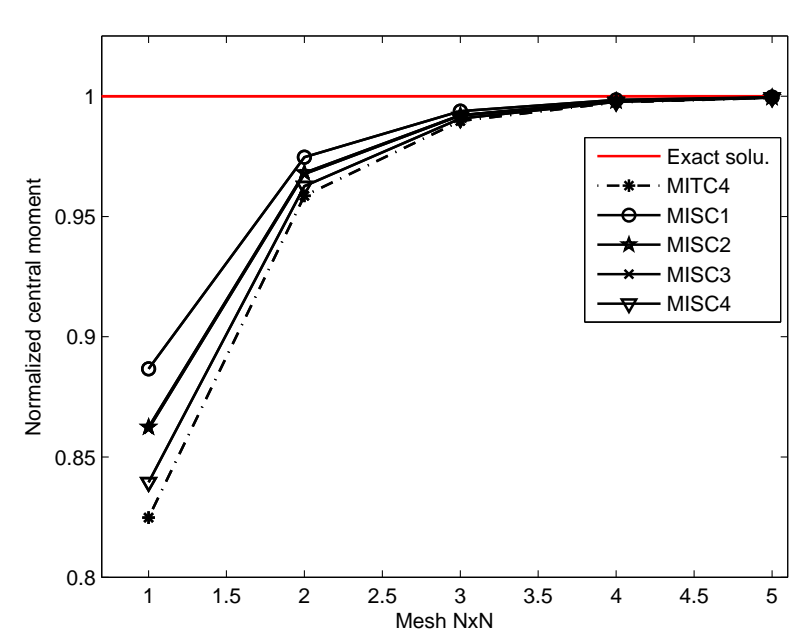

(b)

Figure 8: Normalized deflection and moment at center of clamped square plate subjected to uniform load 


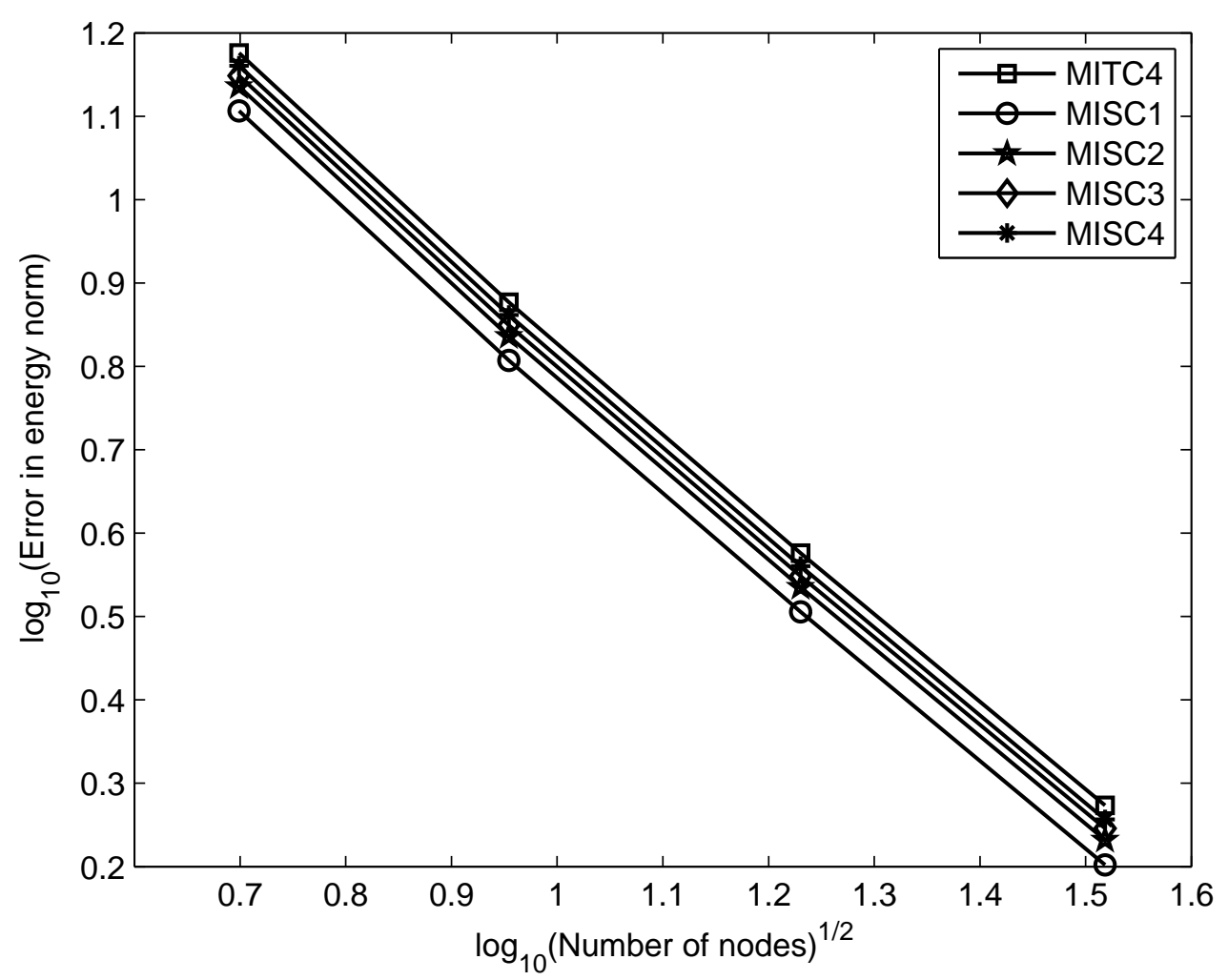

Figure 9: Rate of convergence in energy norm versus with number of nodes for clamped square plate subjected to uniform load 
Table 3: Central deflections $w_{c} /\left(p L^{4} / 100 D\right)$ for the clamped plate subjected to uniform load

\begin{tabular}{|c|c|c|c|c|c|c|c|}
\hline \multirow{2}{*}{$\mathrm{L} / \mathrm{t}$} & \multirow{2}{*}{ elements } & \multicolumn{5}{|c|}{ Mesh } & \multirow[b]{2}{*}{ Exact [51] } \\
\hline & & 2 & 4 & 8 & 16 & 32 & \\
\hline \multirow{5}{*}{10} & MITC4 & 0.1431 & 0.1488 & 0.1500 & 0.1504 & 0.1504 & \multirow{5}{*}{0.1499} \\
\hline & MISC1 & 0.1517 & 0.1507 & 0.1505 & 0.1505 & 0.1505 & \\
\hline & MISC2 & 0.1483 & 0.1500 & 0.1503 & 0.1504 & 0.1505 & \\
\hline & MISC3 & 0.1467 & 0.1496 & 0.1502 & 0.1504 & 0.1504 & \\
\hline & MISC4 & 0.1451 & 0.1493 & 0.1502 & 0.1504 & 0.1504 & \\
\hline \multirow{5}{*}{$10^{2}$} & MITC4 & 0.1213 & 0.1253 & 0.1264 & 0.1267 & 0.1268 & \multirow{5}{*}{0.1267} \\
\hline & MISC1 & 0.1304 & 0.1274 & 0.1269 & 0.1268 & 0.1268 & \\
\hline & MISC2 & 0.1269 & 0.1266 & 0.1267 & 0.1268 & 0.1268 & \\
\hline & MISC3 & 0.1252 & 0.1262 & 0.1266 & 0.1267 & 0.1268 & \\
\hline & MISC4 & 0.1235 & 0.1258 & 0.1265 & 0.1267 & 0.1268 & \\
\hline \multirow{5}{*}{$10^{3}$} & MITC4 & 0.1211 & 0.1251 & 0.1262 & 0.1264 & 0.1265 & \multirow{5}{*}{0.1265} \\
\hline & MISC1 & 0.1302 & 0.1272 & 0.1267 & 0.1266 & 0.1265 & \\
\hline & MISC2 & 0.1266 & 0.1264 & 0.1265 & 0.1265 & 0.1265 & \\
\hline & MISC3 & 0.1249 & 0.1260 & 0.1264 & 0.1265 & 0.1265 & \\
\hline & MISC4 & 0.1233 & 0.1256 & 0.1263 & 0.1265 & 0.1265 & \\
\hline \multirow{5}{*}{$10^{4}$} & MITC4 & 0.1211 & 0.1251 & 0.1262 & 0.1264 & 0.1265 & \multirow{5}{*}{0.1265} \\
\hline & MISC1 & 0.1302 & 0.1272 & 0.1267 & 0.1266 & 0.1265 & \\
\hline & MISC2 & 0.1266 & 0.1264 & 0.1265 & 0.1265 & 0.1265 & \\
\hline & MISC3 & 0.1249 & 0.1260 & 0.1264 & 0.1265 & 0.1265 & \\
\hline & MISC4 & 0.1233 & 0.1256 & 0.1263 & 0.1265 & 0.1265 & \\
\hline \multirow{5}{*}{$10^{5}$} & MITC4 & 0.1211 & 0.1251 & 0.1262 & 0.1264 & 0.1265 & \multirow{5}{*}{0.1265} \\
\hline & MISC1 & 0.1302 & 0.1272 & 0.1267 & 0.1266 & 0.1265 & \\
\hline & MISC2 & 0.1266 & 0.1264 & 0.1265 & 0.1265 & 0.1265 & \\
\hline & MISC3 & 0.1249 & 0.1260 & 0.1264 & 0.1265 & 0.1265 & \\
\hline & MISC4 & 0.1233 & 0.1256 & 0.1263 & 0.1265 & 0.1265 & \\
\hline
\end{tabular}


Table 4: Central moments $M_{c} /\left(p L^{2} / 10\right)$ for the clamped plate subjected to uniform load

\begin{tabular}{|c|c|c|c|c|c|c|c|}
\hline \multirow{2}{*}{$\mathrm{L} / \mathrm{t}$} & \multirow{2}{*}{ elements } & \multicolumn{5}{|c|}{ Mesh } & \multirow[b]{2}{*}{ Exact [51] } \\
\hline & & 2 & 4 & 8 & 16 & 32 & \\
\hline \multirow{5}{*}{10} & MITC4 & 0.1898 & 0.2219 & 0.2295 & 0.2314 & 0.2318 & \multirow{5}{*}{0.231} \\
\hline & MISC1 & 0.2031 & 0.2254 & 0.2304 & 0.2316 & 0.2319 & \\
\hline & MISC2 & 0.1982 & 0.2241 & 0.2300 & 0.2315 & 0.2319 & \\
\hline & MISC3 & 0.1974 & 0.2239 & 0.2300 & 0.2315 & 0.2319 & \\
\hline & MISC4 & 0.1930 & 0.2228 & 0.2297 & 0.2314 & 0.2319 & \\
\hline \multirow{5}{*}{$10^{2}$} & MITC4 & 0.1890 & 0.2196 & 0.2267 & 0.2285 & 0.2289 & \multirow{5}{*}{0.2291} \\
\hline & MISC1 & 0.2031 & 0.2233 & 0.2277 & 0.2287 & 0.2290 & \\
\hline & MISC2 & 0.1976 & 0.2218 & 0.2273 & 0.2286 & 0.2290 & \\
\hline & MISC3 & 0.1974 & 0.2217 & 0.2273 & 0.2286 & 0.2290 & \\
\hline & MISC4 & 0.1923 & 0.2205 & 0.2270 & 0.2286 & 0.2290 & \\
\hline \multirow{5}{*}{$10^{3}$} & MITC4 & 0.1890 & 0.2196 & 0.2267 & 0.2285 & 0.2289 & \multirow{5}{*}{0.2291} \\
\hline & MISC1 & 0.2031 & 0.2233 & 0.2276 & 0.2287 & 0.2290 & \\
\hline & MISC2 & 0.1976 & 0.2218 & 0.2273 & 0.2286 & 0.2289 & \\
\hline & MISC3 & 0.1974 & 0.2217 & 0.2272 & 0.2286 & 0.2289 & \\
\hline & MISC4 & 0.1923 & 0.2205 & 0.2269 & 0.2285 & 0.2289 & \\
\hline \multirow{5}{*}{$10^{4}$} & MITC4 & 0.1890 & 0.2196 & 0.2267 & 0.2285 & 0.2289 & \multirow{5}{*}{0.2291} \\
\hline & MISC1 & 0.2031 & 0.2233 & 0.2276 & 0.2287 & 0.2290 & \\
\hline & MISC2 & 0.1976 & 0.2218 & 0.2273 & 0.2286 & 0.2289 & \\
\hline & MISC3 & 0.1974 & 0.2217 & 0.2272 & 0.2286 & 0.2289 & \\
\hline & MISC4 & 0.1923 & 0.2205 & 0.2269 & 0.2285 & 0.2289 & \\
\hline \multirow{5}{*}{$10^{5}$} & MITC4 & 0.1890 & 0.2196 & 0.2267 & 0.2285 & 0.2289 & \multirow{5}{*}{0.2291} \\
\hline & MISC1 & 0.2031 & 0.2233 & 0.2276 & 0.2287 & 0.2290 & \\
\hline & MISC2 & 0.1976 & 0.2218 & 0.2273 & 0.2286 & 0.2289 & \\
\hline & MISC3 & 0.1974 & 0.2217 & 0.2272 & 0.2286 & 0.2289 & \\
\hline & MISC4 & 0.1923 & 0.2205 & 0.2269 & 0.2285 & 0.2289 & \\
\hline
\end{tabular}




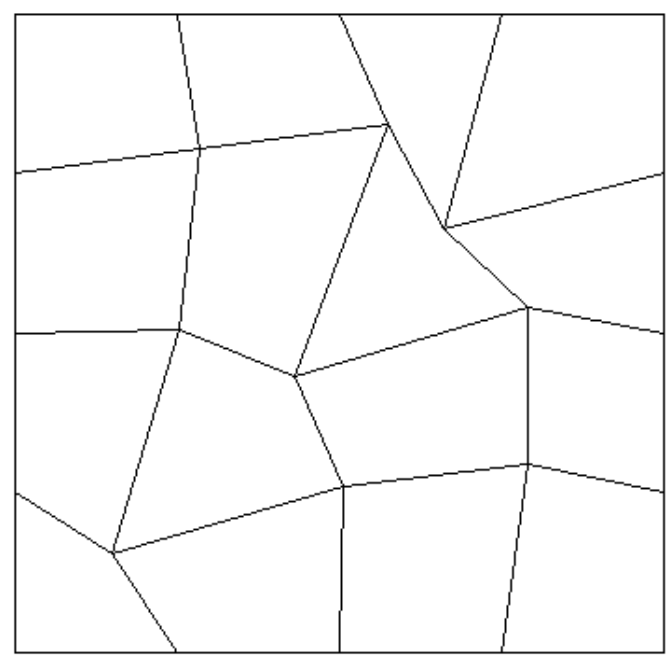

(a)

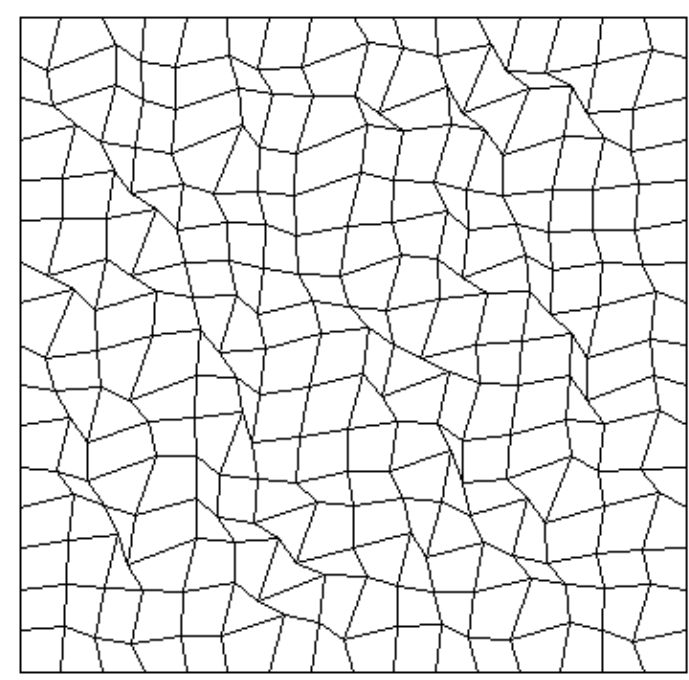

(c)

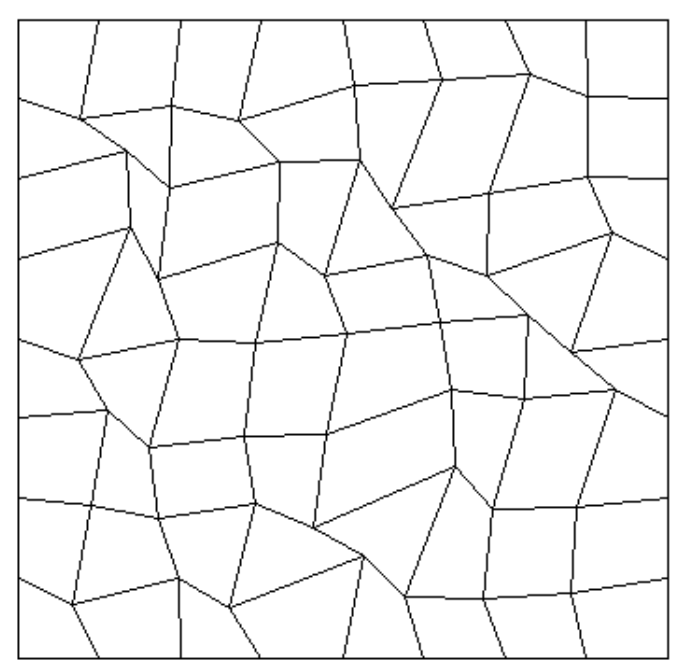

(b)

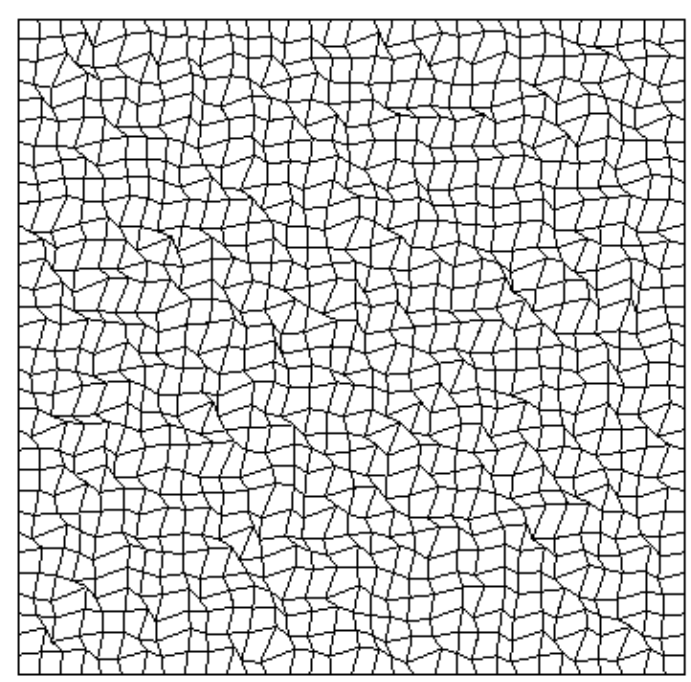

(d)

Figure 10: Analysis of clamped plate with irregular elements: (a) 25; (b) 64; (c) 256; and (d) 1024 


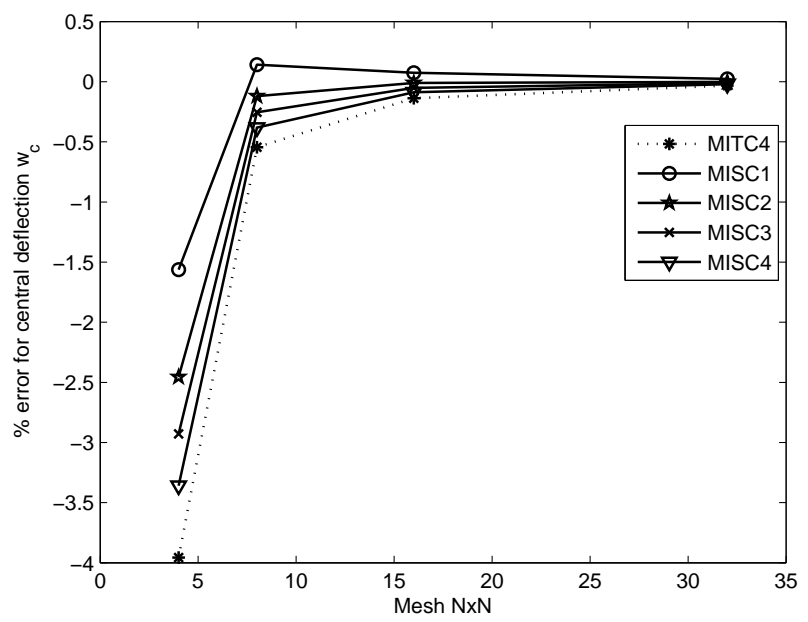

(a)

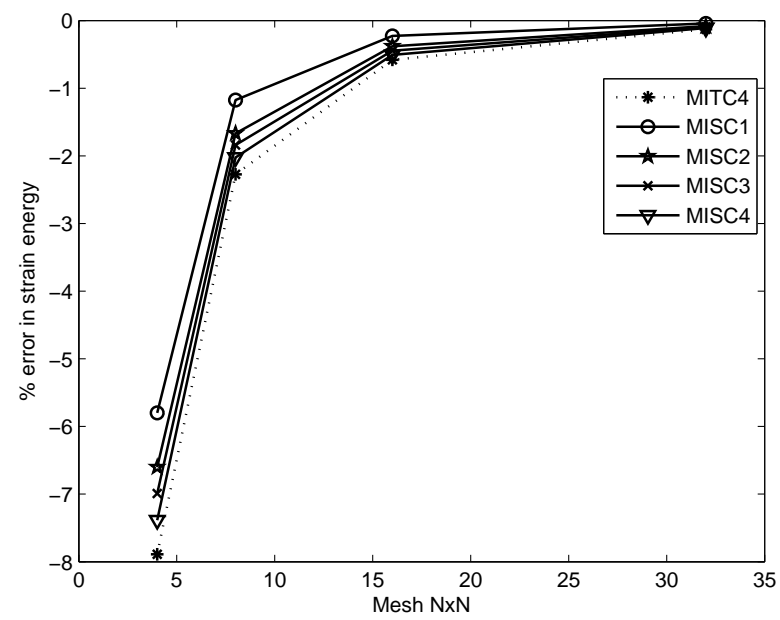

(b)

Figure 11: The convergence test of thin clamped plate ( $\mathrm{t} / \mathrm{L}=0.001$ ) (with irregular elements: (a) the deflection; (b) the strain energy

observed. Exemplarily, we will show the results of the normalized deflection in Figure 14a for the uniform meshes and in Figure 14b for the distorted meshes illustrated in Figure 10.

The numerical results for a simply supported plate subjected to a uniform load are presented in Tables 5-6 and Figures 15 - 16 for a regular mesh. We note that the MISCk elements are more accurate than the MITC4 element but show the same convergence rate in energy. We also see that no shear locking occurs with decreasing thickness. Also, for all elements presented, the displacement results do not seem to be influenced by the value of the thickness ratio, at least in the range $t / L \in\left[10^{-3}, 10^{-5}\right]$. The moments remain accurate throughout the range of thickness ratios that we considered.

\subsection{Skew plate subjected to a uniform load}

\subsubsection{Razzaque's skew plate model.}

Let us consider a rhombic plate subjected to a uniform load $p=1$ as shown in Figure 17a. This plate was originally studied by Razzaque [47]. Dimensions and boundary conditions are specified in Figure 17a, too. Geometry and material parameters are length $L=100$, thickness $t=0.1$, Young's modulus $E=1092000$ and Poisson's ratio $\nu=0.3$. The results in Table 7 show that the accuracy of the presented method is always better than that of the MITC4 element. Figure 18 illustrates the contribution of the von Mises stresses and the level lines for Razzaque's skew plate with our MISC4 element.

\subsubsection{Morley's skew plate model.}

The set-up of a skew plate is shown in Figure 17b. This example was first studied by Morley [43]. The geometry and material parameters are length $L=100$, thickness $t$, Young's modulus $E=1092000$, Poisson's ratio $\nu=0.3$ and a uniform load $p=1$. The values of the deflection at the central point are given in Figure 20 for different plate thickness. The MISCk elements show remarkably good results compared the MITC4 element. The distribution of the von Mises 


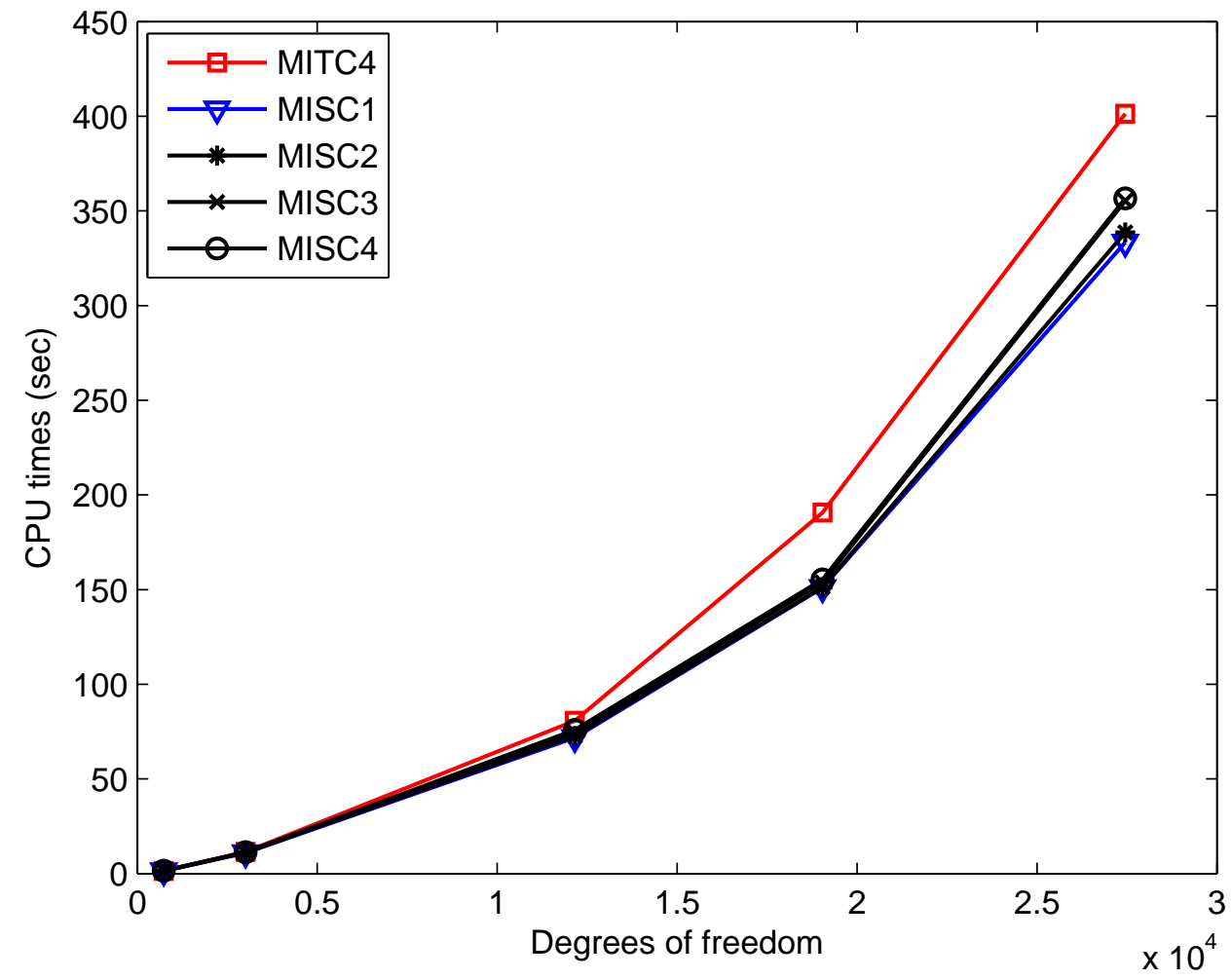

Figure 12: Computational cost for establishing the global stiffness matrix and solving system equations of clamped plate subjected to a uniform load 


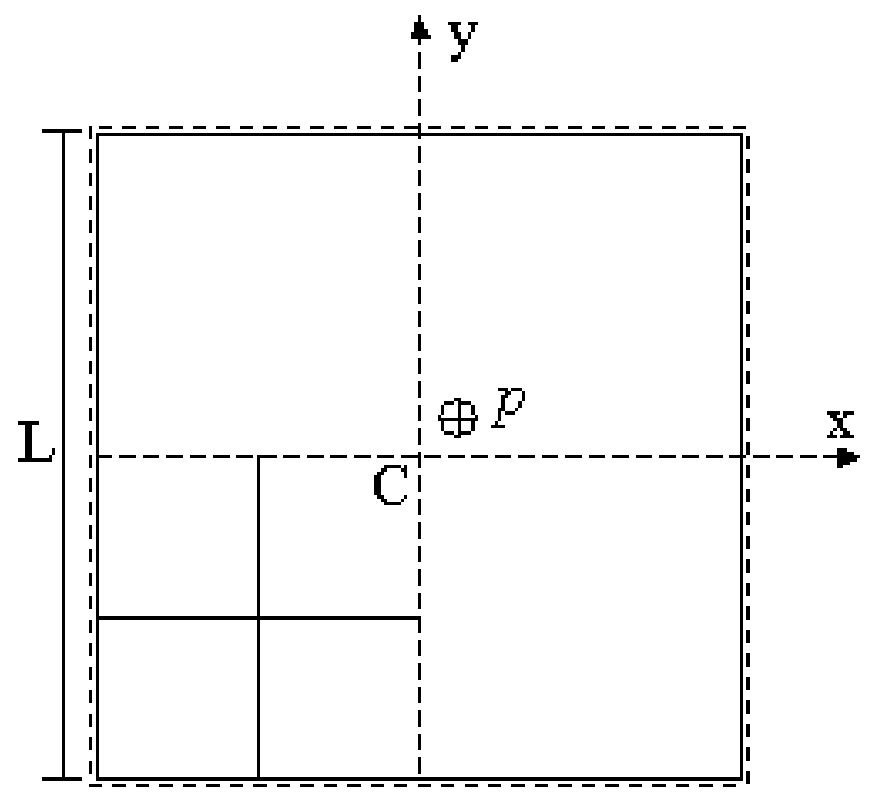

Figure 13: A simply supported square plate subjected to a point load or a uniform load

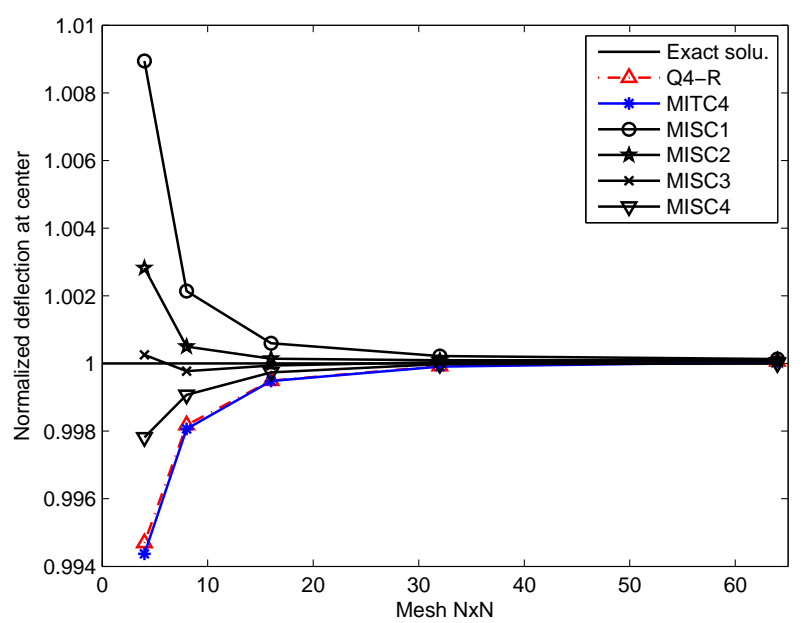

(a) regular mesh

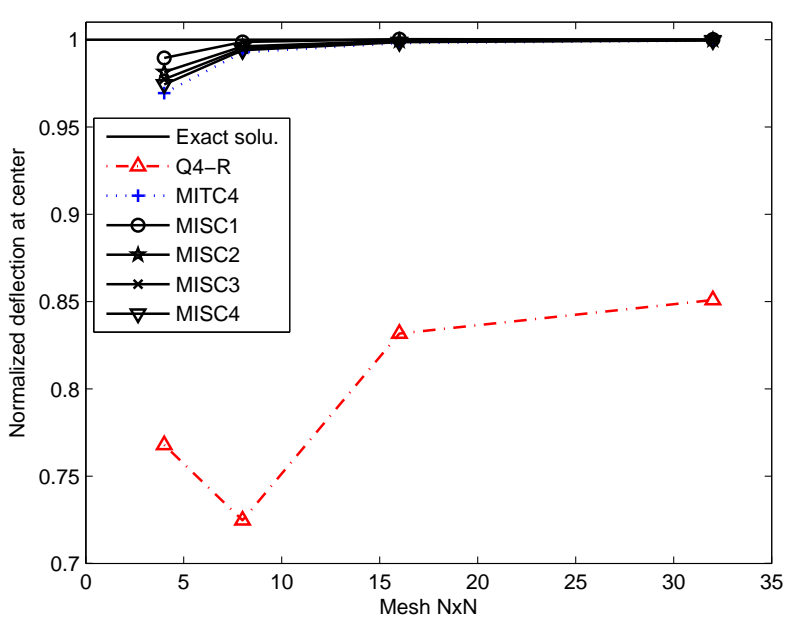

(b) distorted mesh

Figure 14: Normalized deflection at the centre of the simply supported square plate subjected to a center load 
Table 5: Central deflections $w_{c} /\left(p L^{4} / 100 D\right)$ for the simply supported plate subjected to uniform load

\begin{tabular}{|c|c|c|c|c|c|c|c|}
\hline \multirow{2}{*}{$\mathrm{L} / \mathrm{t}$} & \multirow{2}{*}{ elements } & \multicolumn{5}{|c|}{ Mesh } & \multirow[b]{2}{*}{ Exact [51] } \\
\hline & & 2 & 4 & 8 & 16 & 32 & \\
\hline \multirow{5}{*}{10} & MITC4 & 0.4190 & 0.4255 & 0.4268 & 0.4272 & 0.4273 & \multirow{5}{*}{0.4273} \\
\hline & MISC1 & 0.4344 & 0.4290 & 0.4277 & 0.4274 & 0.4273 & \\
\hline & MISC2 & 0.4285 & 0.4277 & 0.4274 & 0.4273 & 0.4273 & \\
\hline & MISC3 & 0.4256 & 0.4270 & 0.4272 & 0.4273 & 0.4273 & \\
\hline & MISC4 & 0.4227 & 0.4263 & 0.4271 & 0.4272 & 0.4273 & \\
\hline \multirow{5}{*}{$10^{2}$} & MITC4 & 0.3971 & 0.4044 & 0.4059 & 0.4063 & 0.4064 & \multirow{5}{*}{0.4064} \\
\hline & MISC1 & 0.4125 & 0.4079 & 0.4068 & 0.4065 & 0.4065 & \\
\hline & MISC2 & 0.4066 & 0.4066 & 0.4065 & 0.4065 & 0.4064 & \\
\hline & MISC3 & 0.4037 & 0.4059 & 0.4063 & 0.4064 & 0.4064 & \\
\hline & MISC4 & 0.4008 & 0.4052 & 0.4062 & 0.4064 & 0.4064 & \\
\hline \multirow{5}{*}{$10^{3}$} & MITC4 & 0.3969 & 0.4041 & 0.4057 & 0.4061 & 0.4062 & \multirow{5}{*}{0.4062} \\
\hline & MISC1 & 0.4123 & 0.4077 & 0.4066 & 0.4063 & 0.4063 & \\
\hline & MISC2 & 0.4064 & 0.4064 & 0.4063 & 0.4062 & 0.4062 & \\
\hline & MISC3 & 0.4035 & 0.4057 & 0.4061 & 0.4062 & 0.4062 & \\
\hline & MISC4 & 0.4006 & 0.4050 & 0.4059 & 0.4062 & 0.4062 & \\
\hline \multirow{5}{*}{$10^{4}$} & MITC4 & 0.3969 & 0.4041 & 0.4057 & 0.4061 & 0.4062 & \multirow{5}{*}{0.4062} \\
\hline & MISC1 & 0.4123 & 0.4077 & 0.4066 & 0.4063 & 0.4063 & \\
\hline & MISC2 & 0.4064 & 0.4064 & 0.4063 & 0.4062 & 0.4062 & \\
\hline & MISC3 & 0.4035 & 0.4057 & 0.4061 & 0.4062 & 0.4062 & \\
\hline & MISC4 & 0.4006 & 0.4050 & 0.4059 & 0.4062 & 0.4062 & \\
\hline \multirow{5}{*}{$10^{5}$} & MITC4 & 0.3969 & 0.4041 & 0.4057 & 0.4061 & 0.4062 & \multirow{5}{*}{0.4062} \\
\hline & MISC1 & 0.4123 & 0.4077 & 0.4066 & 0.4063 & 0.4063 & \\
\hline & MISC2 & 0.4064 & 0.4064 & 0.4063 & 0.4062 & 0.4062 & \\
\hline & MISC3 & 0.4035 & 0.4057 & 0.4061 & 0.4062 & 0.4062 & \\
\hline & MISC4 & 0.4006 & 0.4050 & 0.4059 & 0.4062 & 0.4062 & \\
\hline
\end{tabular}


Table 6: Central moments $M_{c} /\left(p L^{2} / 10\right)$ for the simply supported plate subjected to uniform load

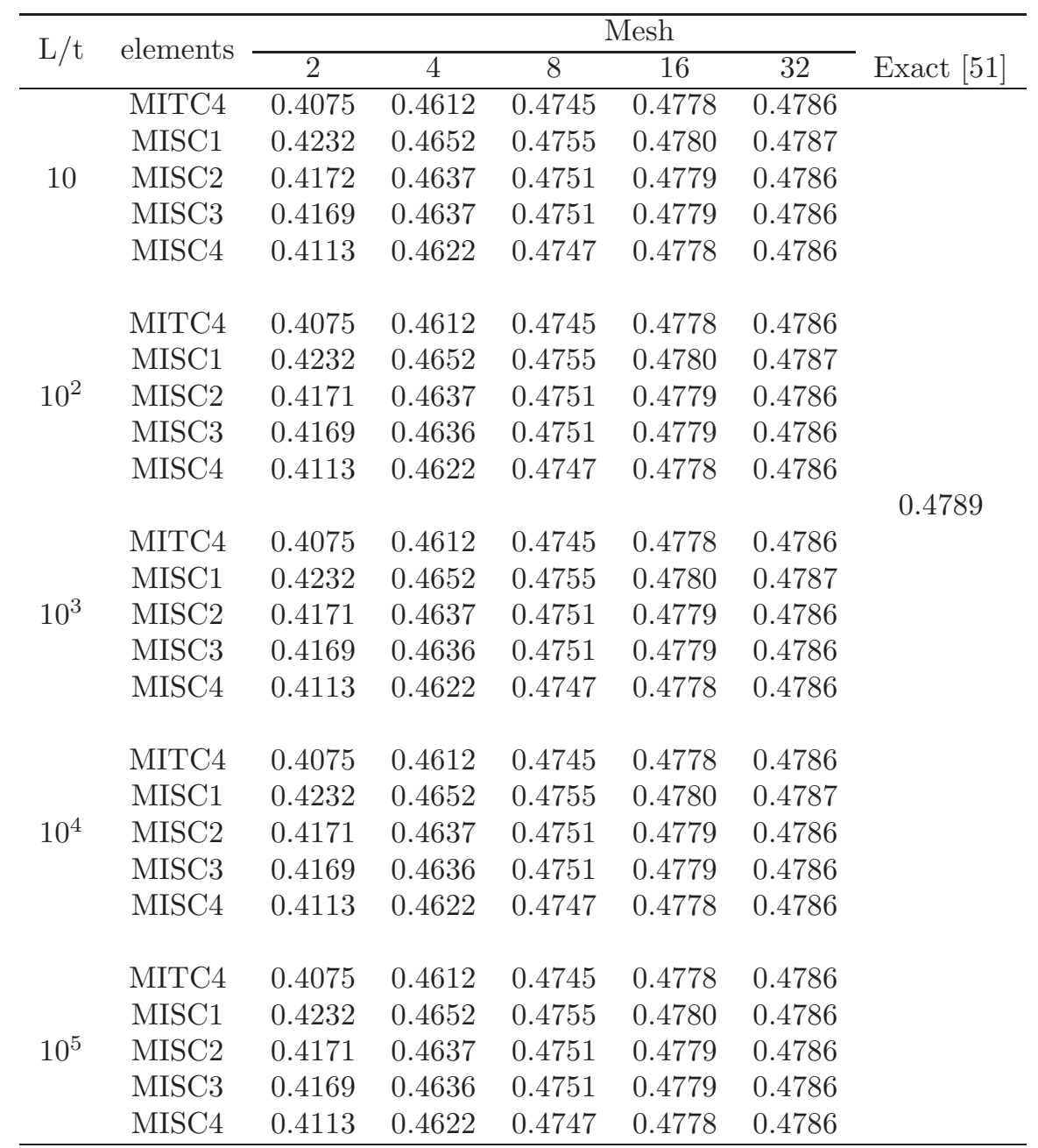




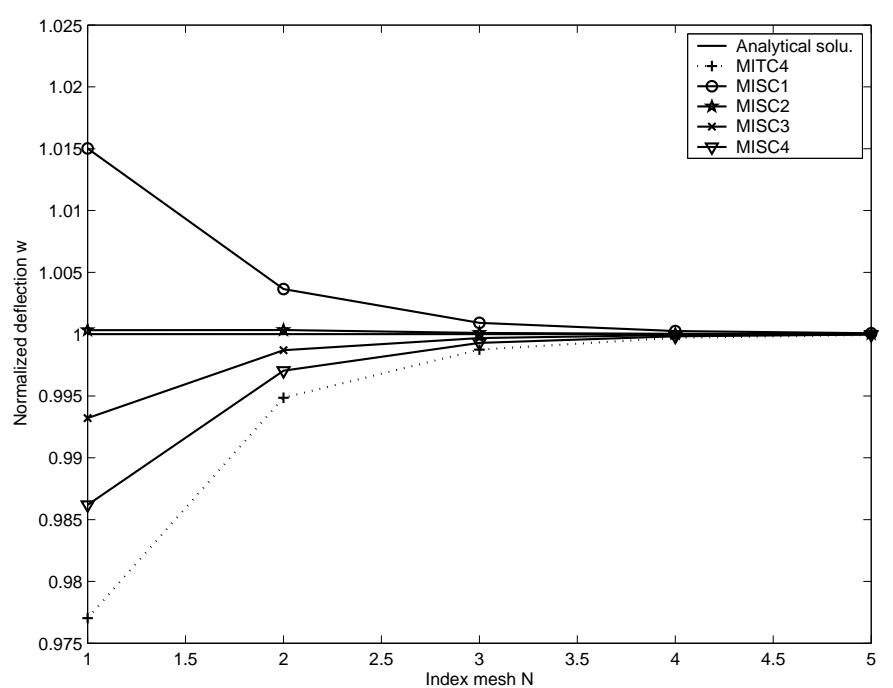

(a)

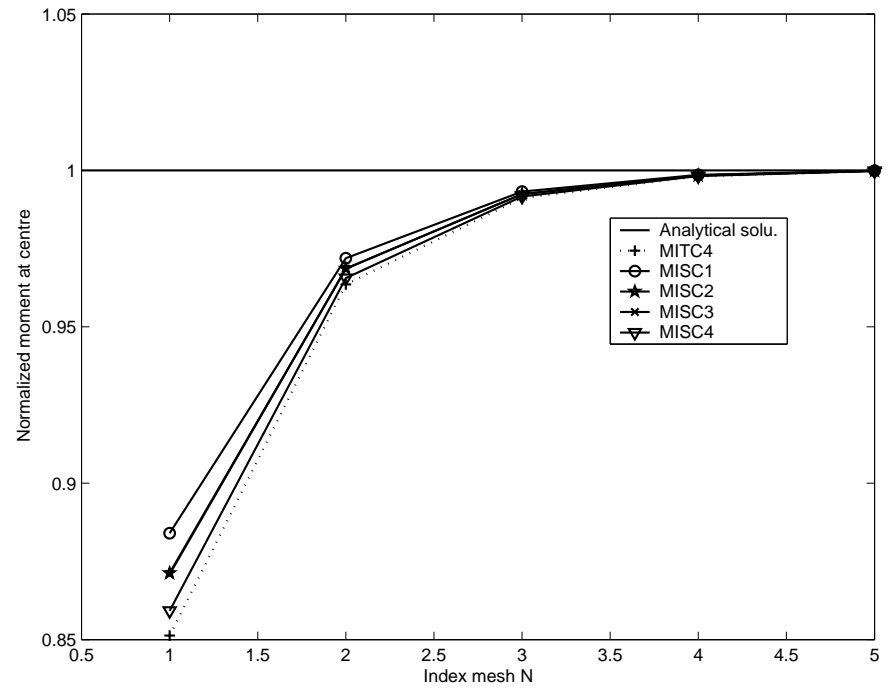

(b)

Figure 15: Normalized deflection and moment at center of simply support square plate subjected to uniform load

Table 7: Central defection and moment of the Razzaque's skew plate

\begin{tabular}{cccccc}
\hline Mesh & MITC4 & MISC1 & MISC2 & MISC3 & MISC4 \\
\hline & & & & & \\
(a) Central deflection $w_{c} / 10^{4}$ & & & & \\
$2 \times 2$ & 0.3856 & 0.3648 & 0.3741 & 0.3781 & 0.3816 \\
$4 \times 4$ & 0.6723 & 0.6702 & 0.6725 & 0.6725 & 0.6724 \\
$6 \times 6$ & 0.7357 & 0.7377 & 0.7377 & 0.7370 & 0.7364 \\
$8 \times 8$ & 0.7592 & 0.7615 & 0.7610 & 0.7604 & 0.7598 \\
$12 \times 12$ & 0.7765 & 0.7781 & 0.7776 & 0.7772 & 0.7769 \\
$16 \times 16$ & 0.7827 & 0.7838 & 0.7834 & 0.7832 & 0.7830 \\
$32 \times 32$ & 0.7888 & 0.7892 & 0.7891 & 0.7890 & 0.7889 \\
Reference $[47,6]$ & & & & & 0.7945 \\
& & & & & \\
(b) Central moment $M_{y} / 10^{3}$ & & & & & \\
$2 \times 2$ & 0.4688 & 0.4688 & 0.4688 & 0.4688 & 0.4688 \\
$4 \times 4$ & 0.8256 & 0.8321 & 0.8301 & 0.8284 & 0.8269 \\
$6 \times 6$ & 0.8976 & 0.9020 & 0.9005 & 0.8994 & 0.8984 \\
$8 \times 8$ & 0.9242 & 0.9272 & 0.9260 & 0.9254 & 0.9245 \\
$12 \times 12$ & 0.9439 & 0.9454 & 0.9448 & 0.9445 & 0.9442 \\
$16 \times 16$ & 0.9510 & 0.9518 & 0.9515 & 0.9513 & 0.9511 \\
$32 \times 32$ & 0.9577 & 0.9580 & 0.9579 & 0.9578 & 0.9578 \\
Reference $[47,6]$ & & & & & 0.9589 \\
\hline & & & & &
\end{tabular}




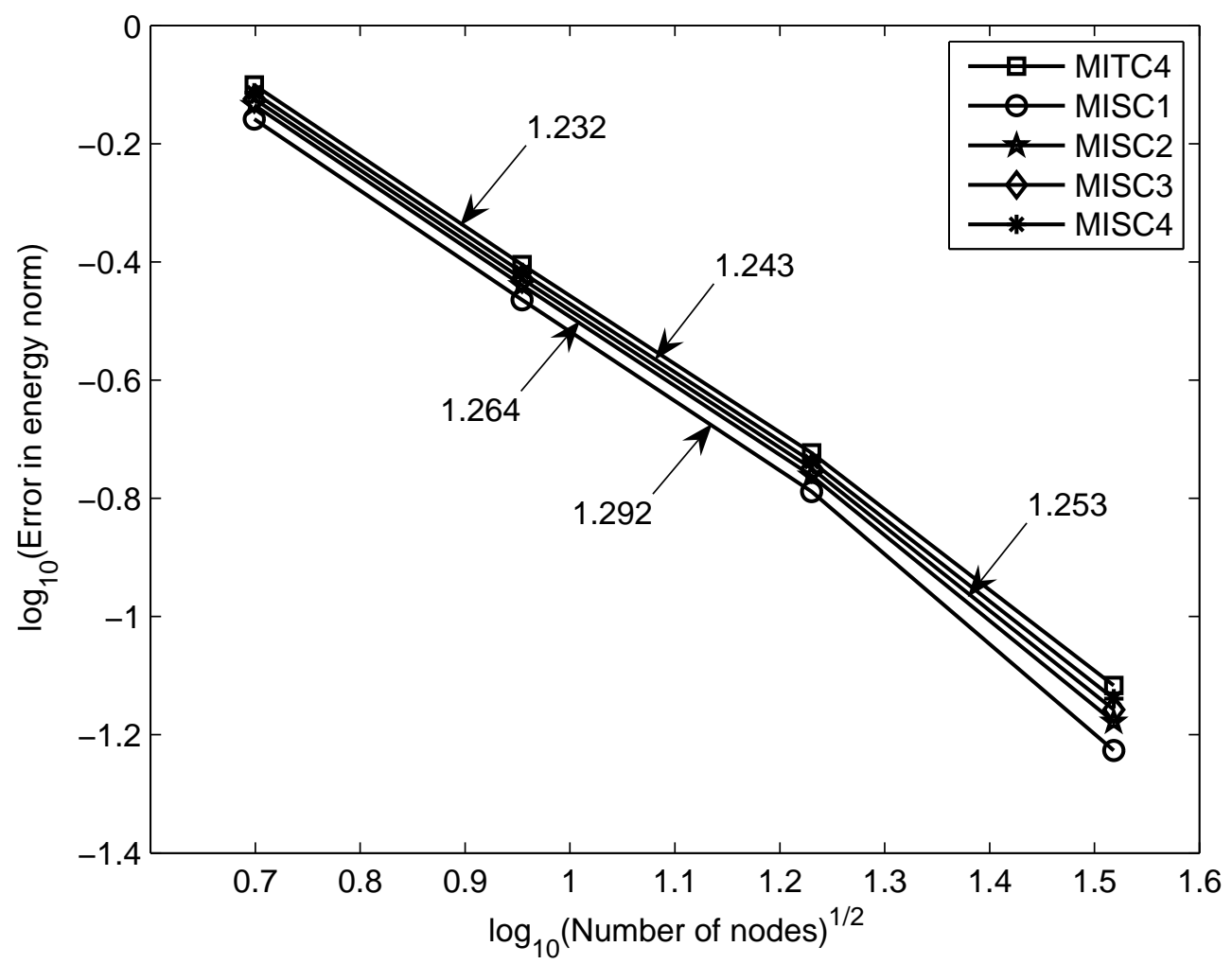

Figure 16: Rate of convergence in energy norm for simply supported square plate subjected to uniform load 


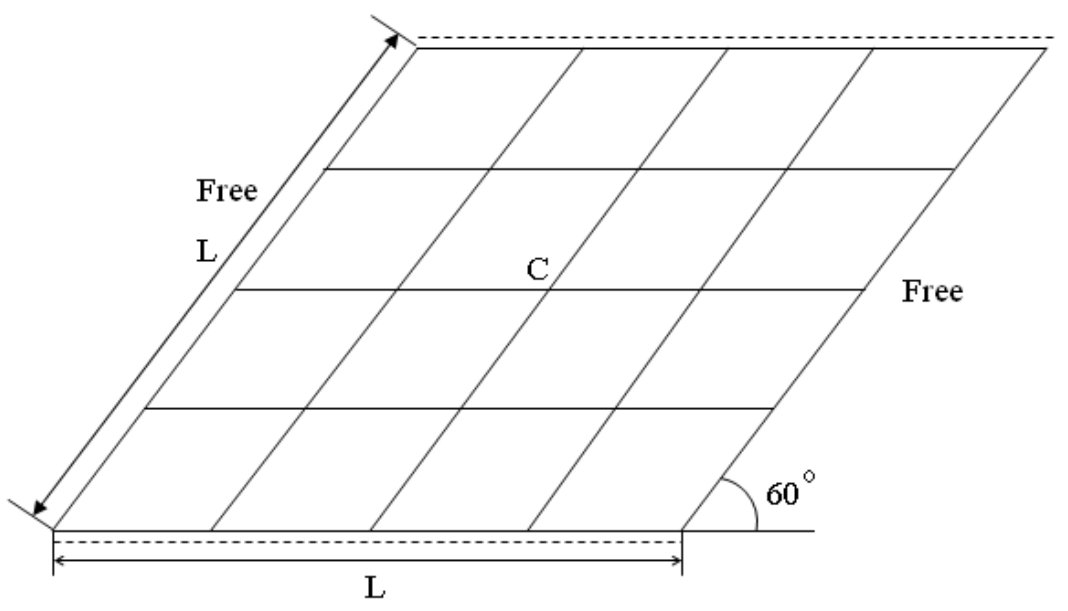

(a)

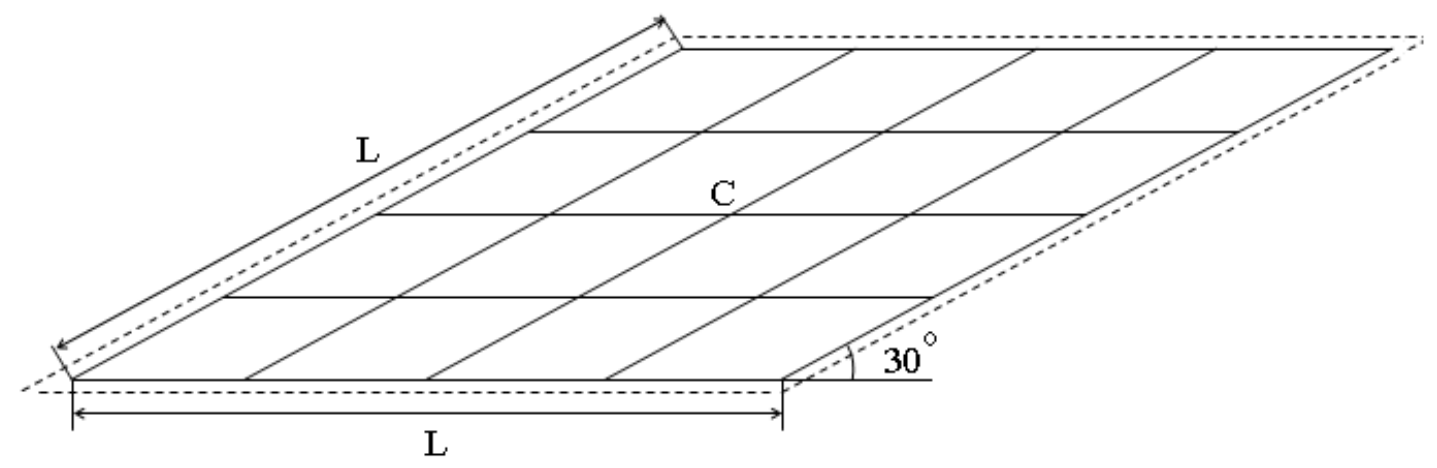

(b)

Figure 17: A simply supported skew plate subjected to a uniform load 


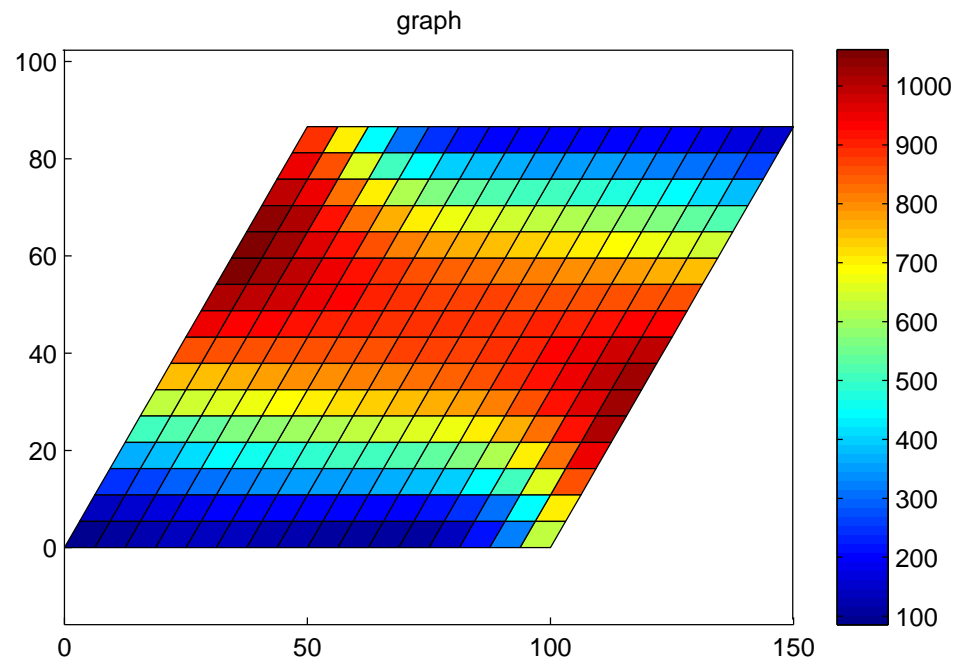

(a)

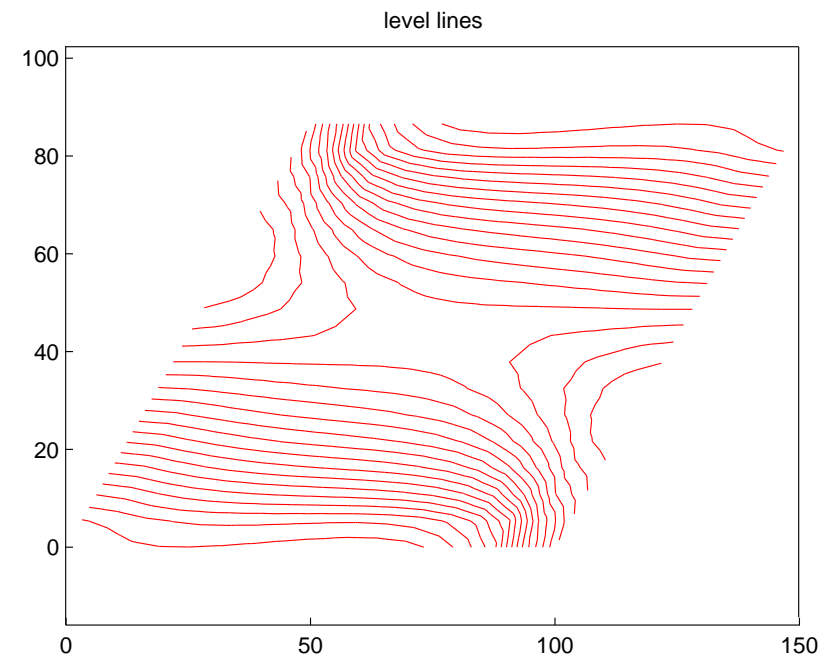

(b)

Figure 18: A distribution of von Mises stress and level lines for Razzaque's skew plate using MISC4 element 
stresses and the level lines are illustrated in Figure 19. It is evident that this problem has the corner singularity. An adaptive approach might be useful for computational reasons.

As we prove mathematically and show numerically in a short communication to be published in Computer Methods in Applied Mechanics and Engineering, in the context of continuum elements, the smoothed finite element method yields solutions comprised between the standard, displacement based FEM and equilibrium, stress based elements. The equilibrium method is recovered when one smoothing cell is used and the displacement method is found in the limit where the number of smoothing cells tends to infinity. It appears like this property could also be verified in the context of plate analysis. At least, the results in Figure 20 tend to indicate that the higher the number of smoothing cells, the closer the solution is to the MITC4 elements, with almost identical behaviour for 4 smoothing cells. On the contrary, it is interesting to note that the one-subcell element (MISC1) overestimates the energy (similarly to equilibrium methods).

\subsection{Corner supported square plate}

Consider a corner supported plate subjected to a uniform load $p=0.03125$ with edge length $L=24$ and thickness $t=0.375$. This example is often studied to test the existence of spurious energy modes. The material parameters are Young's modulus $E=430000$ and Poisson's ratio $\nu=0.38$. The shear correction factor was set to a value of $k=1000$. A symmetric model with an initial mesh of $8 \times 8$ elements is shown in Figure 21. Table 8 shows the convergence of the center deflection. We note that even our rank-deficient MISC1 element gives stable and very accurate results.

We have also carried out a frequency analysis. The mass density is chosen to be $\rho=0.001$ and the normalized frequencies are $\bar{\omega}=\omega L^{2}(D / t \rho)^{-1 / 2}$. The results are illustrated in Table 9 for two mesh densities $(6 \times 6$ and $32 \times 32)$. It can be seen that all proposed elements give stable and accurate solutions.

Table 8: The convergence of center defection for corner supported plate

\begin{tabular}{cccccc}
\hline Elem. per side & \multirow{2}{*}{8} & \multirow{2}{*}{24} & \multirow{2}{*}{48} & 96 \\
\hline DKQ [31] & 0.11914 & 0.11960 & 0.11969 & 0.11974 & 0.11975 \\
G/W [25] & 0.11862 & 0.11947 & 0.11963 & 0.11973 & 0.11975 \\
MITC4 & 0.11856 & 0.11946 & 0.11963 & 0.11973 & 0.11975 \\
MISC1 & 0.11873 & 0.11950 & 0.11965 & 0.11973 & 0.11975 \\
MISC2 & 0.11867 & 0.11949 & 0.11964 & 0.11973 & 0.11975 \\
MISC3 & 0.11864 & 0.11948 & 0.11963 & 0.11973 & 0.11975 \\
MISC4 & 0.11861 & 0.11947 & 0.11963 & 0.11973 & 0.11975 \\
Theory & & & & & 0.12253 \\
\hline
\end{tabular}

\subsection{Clamped circular plate subjected to a concentrated load}

Let us consider a clamped circular plate with radius $R=5$ subjected to a point load $F=1$ at the center. The material and geometric parameters are Young's modulus $E=10.92$, Poisson's ratio $\nu=0.3$ and the thickness of the plate is 1 . The analytical deflection for this problem is

$$
w(r)=\frac{F R^{2}}{16 \pi D}\left(1-\frac{r^{2}}{R^{2}}+\frac{2 r^{2}}{R^{2}} \ln \frac{r}{R}-\frac{8 D}{k G t R^{2}} \ln \frac{r}{R}\right)
$$

A discretization of this problem with 48 elements is illustrated in Figure 22. We exploited the symmetry of the plate and modelled only one quarter. Because of the singularity at the center, the normalized central deflection is evaluated at the radius $r=10^{-3} R$. The numerical results are summarized in Table 10 and Figure 23. The MITC4 and MISCk elements converge to the exact value with refined meshes. However, the convergence in the central deflection is slow 


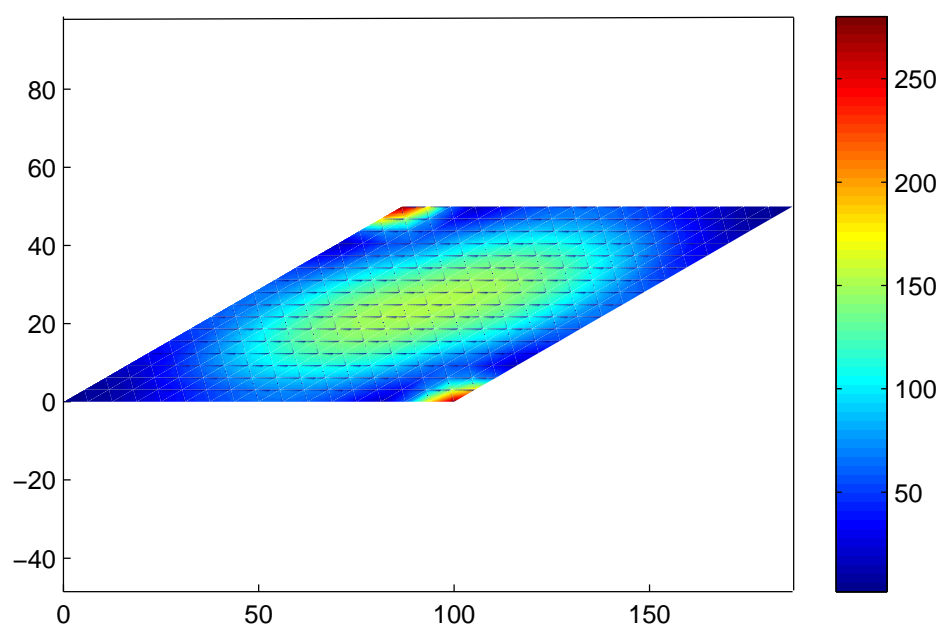

(a)

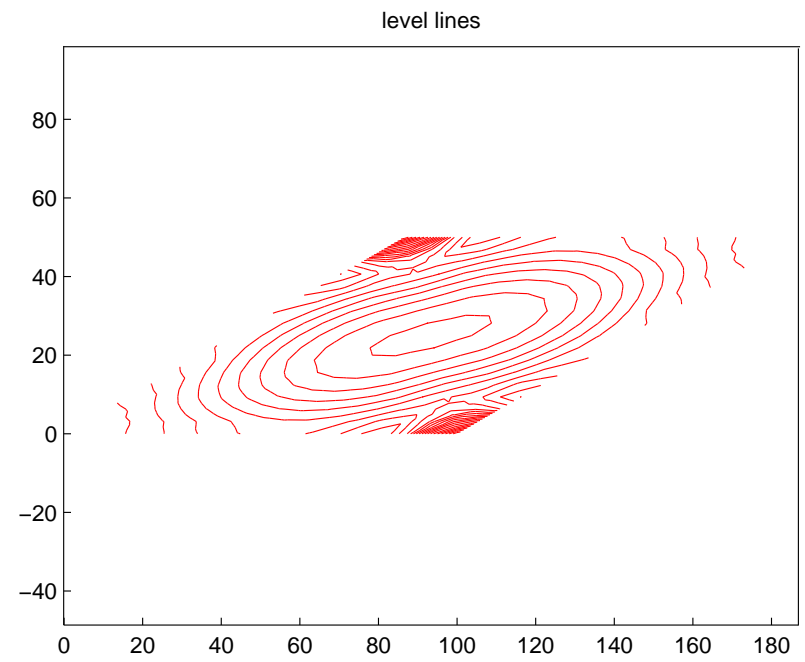

(b)

Figure 19: A distribution of von Mises and level lines for Morley's skew plate using MISC2 element 


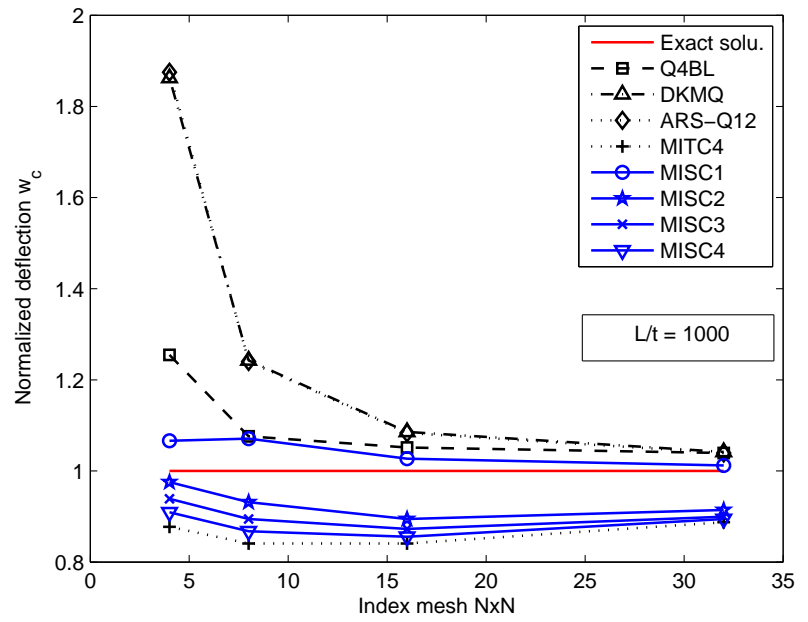

(a)

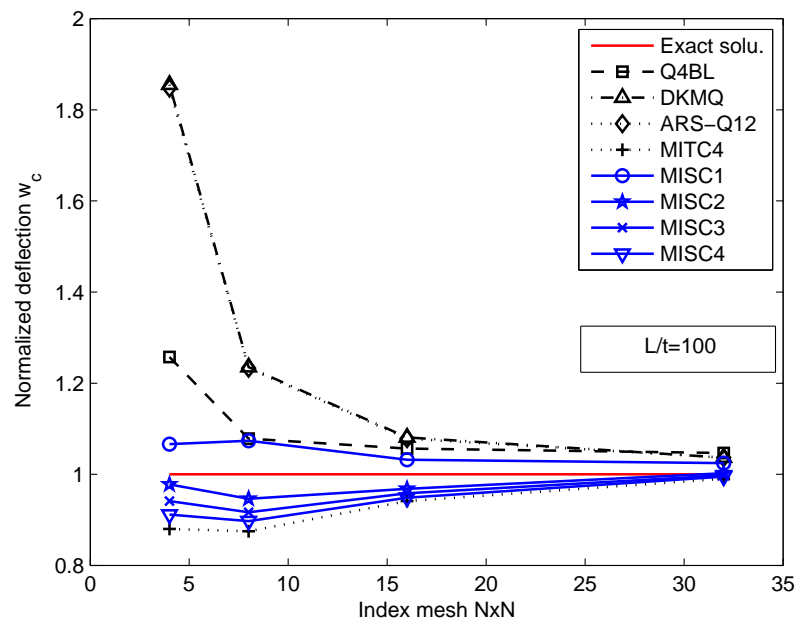

(b)

Figure 20: The convergence of the central deflection $w_{c}$ for Morley plate with different thickness/span ratio 


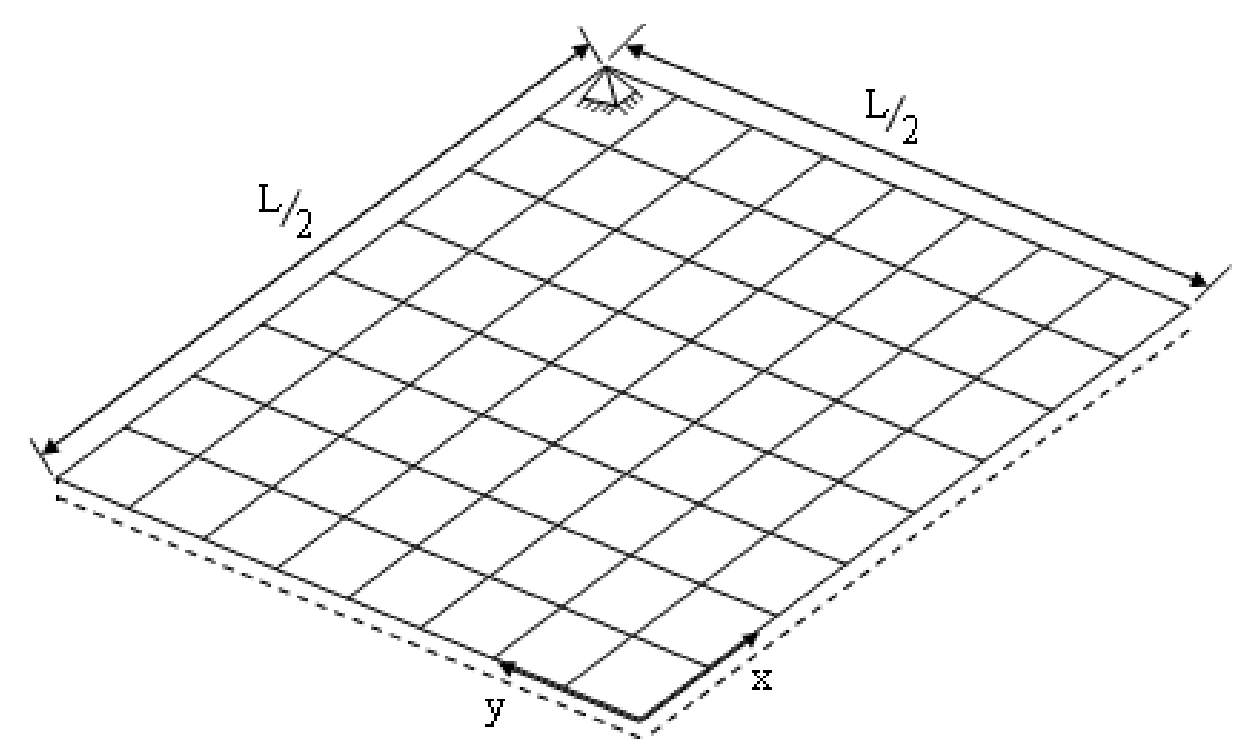

Figure 21: Corner supported plate subjected to uniform load

Table 9: Three lowest frequencies for corner supported plate

\begin{tabular}{ccccccc}
\hline \multirow{2}{*}{ Element } & \multicolumn{3}{c}{$6 \times 6$ mesh } & \multicolumn{3}{c}{$32 \times 32$ mesh } \\
\cline { 2 - 7 } & $\bar{\omega}_{1}$ & $\bar{\omega}_{2}$ & $\bar{\omega}_{3}$ & $\bar{\omega}_{1}$ & $\bar{\omega}_{2}$ & $\bar{\omega}_{3}$ \\
\hline DKQ [31] & 7.117 & 18.750 & 43.998 & - & - & - \\
G/W [25] & 7.144 & 18.800 & 44.105 & - & - & - \\
MITC4 & 7.135 & 18.795 & 44.010 & 7.036 & 18.652 & 43.163 \\
MISC1 & 7.136 & 18.799 & 44.011 & 7.075 & 18.661 & 43.553 \\
MISC2 & 7.141 & 18.800 & 44.065 & 7.075 & 18.661 & 43.555 \\
MISC3 & 7.143 & 18.800 & 44.092 & 7.075 & 18.661 & 43.556 \\
MISC4 & 7.145 & 18.800 & 44.119 & 7.076 & 18.661 & 43.557 \\
Theory [36] & & & & 7.120 & 19.600 & 44.400 \\
\hline
\end{tabular}


due to the singularity at the center. To increase the convergence rate of the problem, an adaptive local refinement procedure should be considered in the future. If the ratio $r / R$ is large enough, the numerical results are very close to the analytical solution.

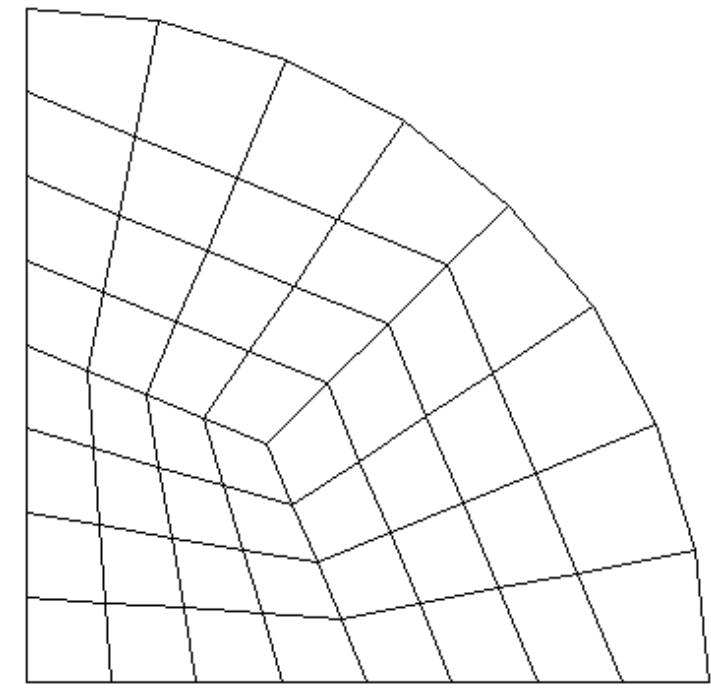

Figure 22: Clamped circular plate subjected to concentrated load

Table 10: The normalized defection at center for circular plate

\begin{tabular}{cccccc}
\hline Mesh & 2 & 4 & 8 & 16 & 32 \\
\hline MITC4 & 0.7817 & 0.8427 & 0.8874 & 0.9278 & 0.9671 \\
MISC1 & 0.8011 & 0.8492 & 0.8893 & 0.9284 & 0.9673 \\
MISC2 & 0.7910 & 0.8457 & 0.8883 & 0.9281 & 0.9672 \\
MISC3 & 0.7880 & 0.8448 & 0.8880 & 0.9280 & 0.9672 \\
MISC4 & 0.7854 & 0.8439 & 0.8877 & 0.9279 & 0.9672 \\
\hline
\end{tabular}

\section{$5 \quad$ Closure and opening}

A quadrilateral plate element based on a mixed interpolation with smoothed curvatures has been proposed. Except for the MISC1 element that exhibits two zero energy modes, the MISC2, MISC3 and MISC4 elements maintain a sufficient rank and no zero energy modes are present. Moreover, all proposed elements do not exhibit shear locking in the limit to thin plates. It is also shown that the MISCk element passes the patch test. In comparison to the well known MITC4 element, the proposed elements are slightly more accurate ${ }^{3}$ for regular meshes and especially perform better than for irregular meshes or coarse meshes while their computational cost is lower.

The element with the best performance is the MISC1 element but it exhibits two zero energy modes. However, for the examples tested here, no instabilities were observed. The elimination of the zero-energy modes of our MISC1 elements will be investigated in the future. The MISC2 element is almost of the same accuracy as the MISC1 element and it is stable but it is slower.

${ }^{3}$ for all examples tested 


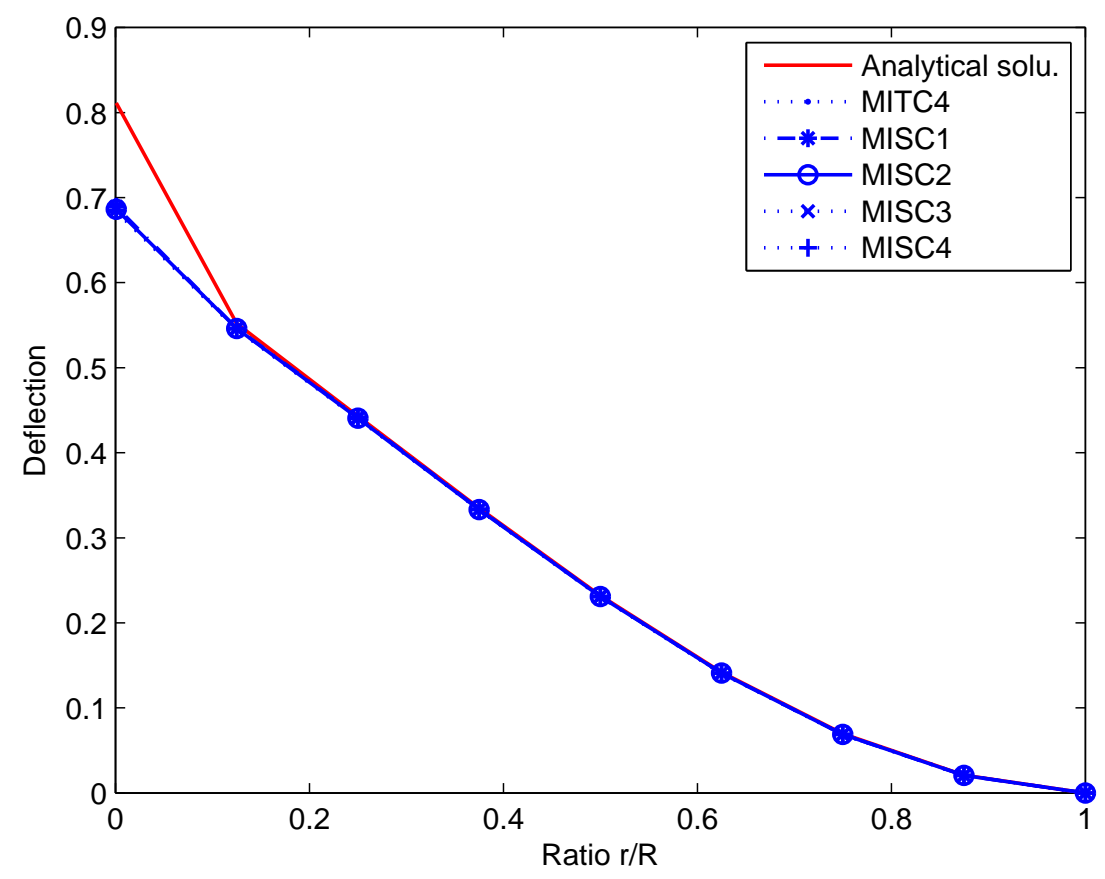

Figure 23: Clamped circular plate subjected to concentrated load

We believe that our element is especially useful for certain types of problems where locally large deformations or strains occur, e.g. ductile cracking where crack initiation and propagation occurs under large strains and large deformation. It is important to retain accuracy in a local region before cracking happens in order to obtain the correct crack path $[46,16]$. This will be investigated in the future using open source XFEM libraries $[15,21]$. The MISCk elements can be extended to non-linear material models including strong discontinuities such as in the eXtended finite element method (XFEM) introduced by $[8,9,42,41]$ and improved to handle complex industrial structures in $[14,11]$ and, later, $[59,58,56,57]$. For discontinuous problems, an adaptive procedure might be useful as well, for instance, following the seminal work presented in $[13,12,20]$. We expect the $h$ - adaptivity procedure to be simplified compared to standard finite element formulations, because remeshing will not be as severely constrained by mesh quality requirements as in standard finite element methods.

Another study will concern the shear term. By replacing $2 \times 2$ Gauss integration on the shear term with a reduced integration with stabilization, we expect the element to be even better suited to handle arbitrary mesh distortions. 


\section{References}

[1] ABAQUS/Standard User's Manual,Version 6.4. Hibbitt, Karlsson and Sorensen, Inc.: Rawtucket, Rhode Island, 2004.

[2] F. Armero and D. Ehrlich. Finite element methods for the multi-cale modeling of softening hinge lines in plates at failure.

[3] K.J. Bathe. Finite element procedures. Englewood Cliffs, NJ: Prentice-Hall, Masetchuset(MIT), 1996.

[4] K.J. Bathe and E.N. Dvorkin. A four-node plate bending element based on Mindlin/Reissener plate theory and a mixed interpolation. International Journal for Numerical Methods in Engineering, 21:367 - 383, 1985.

[5] K.J. Bathe and E.N. Dvorkin. A formulation of general shell elements. the use of mixed interpolation of tensorial components. International Journal for Numerical Methods in Engineering, 22:697 - 722, 1986.

[6] J.L. Batoz and G. Dhatt. Modélisation des structures par éléments finis, Vol.2, poutres et plaques. Hermès, 1990.

[7] G.P. Bazeley, Y.K. Cheung, B.M. Irons, and O.C. Zienkiewicz. Triangular elements in plate bending. Proc. First Conf. on Matrix Methods in Stuctural Mechanics, Wright-Patterson AFB, Ohio, 1965.

[8] T. Belytschko and T. Black. Elastic crack growth in finite elements with minimal remeshing. International Journal for Numerical Methods in Engineering, 45(5):601-620, 1999.

[9] T. Belytschko, N. Moes, S. Usui, and C. Parimi. Arbitrary discontinuities in finite elements. International Journal for Numerical Methods in Engineering, 50(4):993-1013, 2001.

[10] K. Bletzinger, M. Bischoff, and E. Ramm. A unified approach for shear-locking-free triangular and rectangular shell finite elements. Computers and Structures, 75:321-334, 2000.

[11] S. Bordas, J.G. Conley, B. Moran, J. Gray, and E. Nichols. A simulation-based design paradigm for complex cast components. Engineering with Computers, 23(1):25-37, 2007.

[12] S. Bordas and M. Duflot. Derivative recovery and a posteriori error estimation in extended finite element methods. Computer Methods in Applied Mechanics and Engineering, 2007. in press, http://dx.doi.org/10.1016/j.cma.2007.03.011.

[13] S. Bordas, M. Duflot, and P. Le. A simple a posteriori error estimator for the extended finite element method. Communications in Numerical Methods in Engineering, 2007. in press, doi:10.1002/cnm.1001.

[14] S. Bordas and B. Moran. Enriched finite elements and level sets for damage tolerance assessment of complex structures. Engineering Fracture Mechanics, 73:1176-1201, 2006.

[15] S. Bordas, V.P. Nguyen, C. Dunant, H. Nguyen-Dang, and A. Guidoum. An extended finite element library. International Journal for Numerical Methods in Engineering, 2007. 10.1002/nme.1966.

[16] S. Bordas, T. Rabczuk, and G. Zi. Three-dimensional crack initiation, propagation, branching and junction in non-linear materials by an extended meshfree method without asymptotic enrichment. Engineering Fracture Mechanics, 2007. in press.

[17] J.S. Chen, C.T. Wu, S. Yoon, and Y. You. A stabilized conforming nodal integration for Galerkin mesh-free methods. International Journal for Numerical Methods in Engineering, 50:435-466, 2001.

[18] W. Chen and Y.K. Cheung. Refined quadrilateral element based on Mindlin/Reissner plate theory. International Journal for Numerical Methods in Engineering, 47:605-627, 2000.

[19] C. Chinosi, C. Lovadina, and L.D. Marini. Nonconforming locking-free finite elements for Reissner-Mindlin plates. Computer Methods in Applied Mechanics and Engineering, 195:3448-3460, 2006.

[20] M. Duflot and S. Bordas. An extended global recovery procedure for a posteriori error estimation in extended finite element methods. International Journal for Numerical Methods in Engineering, in press. 
[21] C. Dunant, P. Nguyen, M. Belgasmia, S. Bordas, A. Guidoum, and H. Nguyen-Dang. Architecture trade-offs of including a mesher in an object-oriented extended finite element code. European journal of mechanics, (16):237-258, 2007. special issue on the extended finite element method.

[22] E.N. Dvorkin and K.J. Bathe. A continuum mechanics based four-node shell element for general nonlinear analysis. Engineering Computations, 1994.

[23] B. Fraeijs de Veubeke and G. Sander. An equilibrium model for plate bending. International Journal of Solids and Structures, Pergamon Press:447-468, London 1968.

[24] O. Garcia, E.A. Fancello, C.S. Barcellos, and C.A. Duarte. Hp clouds in Mindlin's thick plate model. International Journal for Numerical Methods in Engineering, 47:1381-1400, 2000.

[25] F. Gruttmann and W. Wagner. A stabilized one-point integrated quadrilateral Reissener-Mindlin plate element. International Journal for Numerical Methods in Engineering, 61:2273 - 2295, 2004.

[26] T.J.R. Hughes. The Finite Element Method. Prentice-Hall, Englewood Cliffs, NJ, 1987.

[27] T.J.R. Hughes, M. Cohen, and M. Haroun. Reduced and selective integration techniques in finite element method of plates. Nuclear Engineering Design, 46:203 - 222, 1978.

[28] T.J.R. Hughes, R.L. Taylor, and W. Kanoknukulchai. Simple and efficient element for plate bending. International Journal for Numerical Methods in Engineering, 11:1529 - 1543, 1977.

[29] T.J.R. Hughes and T. Tezduyar. Finite elements based upon Mindlin plate theory with particular reference to the four-node isoparametric element. Journal of Applied Mechanics, 1981.

[30] D. D. Jensen and K. C. Park. Equilibrium constrained assumed natural co-ordinate strain plate elements. IJNME, 38:2951-2977, 1995.

[31] J.L.Batoz and M.B.Tahar. Evaluation of a new quadrilateral thin plate bending element. International Journal for Numerical Methods in Engineering, 18:1655-1677, 1982.

[32] P. Krysl and T. Belytschko. Analysis of thin plates by the element-free Galerkin method. Computational Mechanics, 16:1-10, 1995.

[33] P. Krysl and T. Belytschko. Analysis of thin shells by the element-free Galerkin method. International Journal for Numerical Methods in Engineering, 33:3057-3080, 1996.

[34] S.W. Lee and T.H.H. Pian. Improvement of plate and shell finite element by mixed formulation. AIAA Journal, 16:29-34, 1978.

[35] S.W. Lee and C. Wong. Mixed formulation finite elements for Mindlin theory plate bending. International Journal for Numerical Methods in Engineering, 18:1297-1311, 1982.

[36] A.W Leissa. Vibration of plates. NASA SP-160, 1969.

[37] Q. Li, J. Soric, T. Jarak, and S.N. Atluri. A locking-free meshless local Petrov-Galerkin formulation for thick and thin plates. Jounal of Computational Physics, 208:116-133, 2005.

[38] G.R. Liu, K.Y. Dai, and T.T. Nguyen. A smoothed finite element for mechanics problems. Computational Mechanics, 39:859-877, 2007.

[39] G.R. Liu, T.T. Nguyen, K.Y. Dai, and K.Y. Lam. Theoretical aspects of the smoothed finite element method (sfem). International Journal for Numerical Methods in Engineering, in press, 2006.

[40] I.W. Liu and C. C. Lin. A new conforming quadrilateral plate bending element. IJNME, 36:2921-2937, 1993.

[41] N Moes and T. Belytschko. Extended finite element method for cohesive crack growth. Engineering Fracture Mechanics, 69:813-834, 2002.

[42] N. Moes, J. Dolbow, and T. Belytschko. A finite element method for crack growth without remeshing. International Journal for Numerical Methods in Engineering, 46(1):133-150, 1999.

[43] L.S.D. Morley. Skew plates and structures. Pergamon Press: Oxford, 1963. 
[44] X.H. Nguyen, S. Bordas, and H. Nguyen-Dang. Smooth strain finite elements : selective integration. Collection of papers from Prof. Nguyen-Dang Hung's former students, Vietnam National University -HCM Publishing House:88 - 106, 2006.

[45] T.H.H. Pian and P. Tong. Basis of finite elements for solids continua. International Journal for Numerical Methods in Engineering, 1:3-28, 1969.

[46] T. Rabczuk, S. Bordas, and G. Zi. A three-dimensional meshfree method for continuous crack initiation, nucleation and propagation in statics and dynamics. Computational Mechanics, 2007. doi:10.1007/s00466-006-0122-1.

[47] A. Razzaque. Program for triangular bending elements with derivative smoothing. International Journal for $N u$ merical Methods in Engineering, 6:333-345, 1973.

[48] J.C. Simo and T.J.R. Hughes. On the variational foundation of assumed strain methods. ASME Journal of Applied Mechanics, 53:51 - 54, 1986 .

[49] J.C. Simo and M.S. Rifai. A class of mixed assumed strain methods and the method of incompatible modes. International Journal for Numerical Methods in Engineering, 29:1595 - 1638, 1990.

[50] J. Soric, Q. Li, T. Jarak, and S.N. Atluri. The meshless local Petrov-Galerkin (MLPG) formulation for analysis of thick plates. Computer Modeling in Engineering and Sciences, 6:349-357, 2004.

[51] R.L. Taylor and F. Auricchio. Linked interpolation for Reissner-Mindlin plate elements. Part I-a simple triangle. International Journal for Numerical Methods in Engineering, 36:3056-3066, 1993.

[52] D. Wang and J.S. Chen. Locking -free stabilized conforming nodal integration for meshfree Mindlin-Reissner plate formulation. Computer Methods in Applied Mechanics and Engineering, 193:1065-1083, 2004.

[53] D. Wang, S.B. Dong, and J.S. Chen. Extended meshfree analysis of transverse and inplane loading of a laminated anisotropic plate of general planform geometry. International Journal of Solids and Structures, 43:144-171, 2006.

[54] K. Washizu. Variational Methods in Elasticity and Plasticity(3rd edn). Pergamon Press, New York, 1982.

[55] S.L. Weissman and R.L. Taylor. Resultant fields for mixed plate bending elements. Computer Methods in Applied Mechanics and Engineering, 79:321-355, 1990.

[56] E. Wyart. Three-dimensional crack analysis in aeronautical structures using the Substructured Finite Element/Extended Finite Element Method. PhD thesis, Université catholique de Louvain, 2007.

[57] E. Wyart, D. Coulon, M. Duflot, T. Pardoen, J.-F. Remacle, and F. Lani. A substructured fe shell/xfe 3d method for crack analysis. accepted, 2007.

[58] E. Wyart, D. Coulon, P. Martiny, T. Pardoen, J.-F. Remacle, and F. Lani. A substructured fe/xfe method for stress intensity factors computation in an industrial structure. Revue Européenne de Mécanique Numérique, in press, 2007.

[59] E. Wyart, M. Duflot, D. Coulon, P. Martiny, T. Pardoen, J.-F. Remacle, and F. Lani. Substructuring fe-xfe approaches applied to three dimensional crack propagation. Journal of Computational and Applied Mathematics, in press, 2007.

[60] J.W. Yoo, B. Moran, and J.S. Chen. Stabilized conforming nodal integration in the natural-element method. International Journal for Numerical Methods in Engineering, 60:861-890, 2004.

[61] O. C. Zienkiewicz, L. F. Zeng Z. Xu, A. Samuelsson, and N. E. Wiberg. Linked interpolation for Reissner - Mindlin plate elements: Part i-a simple quadrilateral. IJNME, 36:3043-3056, 1993.

[62] O.C. Zienkiewicz and R.L. Taylor. The Finite Element Method. 5th Edition, Butterworth Heinemann, Oxford, 2000.

[63] O.C. Zienkiewicz, R.L. Taylor, and J.M. Too. Reduced intgration technique in general analysis of plates and shells simple and efficient element for plate bending. International Journal for Numerical Methods in Engineering, $3: 275-290,1971$. 